\title{
Advanced Mobility Handover for Mobile IPv6 Based Wireless Networks
}

\author{
Ali Safa Sadiq, ${ }^{1}$ Norsheila Binti Fisal, ${ }^{2}$ Kayhan Zrar Ghafoor, ${ }^{3}$ and Jaime Lloret ${ }^{4}$ \\ ${ }^{1}$ Faculty of Computer Systems and Software Engineering, Universiti Malaysia Pahang, Lebuhraya Tun Razak, \\ Gambang, 26300 Kuantan, Pahang, Malaysia \\ ${ }^{2}$ UTM MIMOS CoE in Telecommunication Technology Faculty of Electrical Engineering, Universiti Teknologi Malaysia (UTM), \\ 81310 Johor Bahru, Johor, Malaysia \\ ${ }^{3}$ Faculty of Engineering, University of Koya, Daniel Miterrand Boulevard, Koya KOY45, Kurdistan, Iraq \\ ${ }^{4}$ Instituto de Investigacion para la Gestion Integrada de Zonas Costeras, Universidad Politecnica de Valencia, 46022 Valencia, Spain
}

Correspondence should be addressed to Norsheila Binti Fisal; sheila@fke.utm.my

Received 5 August 2014; Accepted 17 November 2014; Published 31 December 2014

Academic Editor: Weifeng Sun

Copyright (C) 2014 Ali Safa Sadiq et al. This is an open access article distributed under the Creative Commons Attribution License, which permits unrestricted use, distribution, and reproduction in any medium, provided the original work is properly cited.

\begin{abstract}
We propose an Advanced Mobility Handover scheme (AMH) in this paper for seamless mobility in MIPv6-based wireless networks. In the proposed scheme, the mobile node utilizes a unique home IPv6 address developed to maintain communication with other corresponding nodes without a care-of-address during the roaming process. The IPv6 address for each MN during the first round of AMH process is uniquely identified by HA using the developed MN-ID field as a global permanent, which is identifying uniquely the IPv6 address of MN. Moreover, a temporary MN-ID is generated by access point each time an MN is associated with a particular AP and temporarily saved in a developed table inside the AP. When employing the AMH scheme, the handover process in the network layer is performed prior to its default time. That is, the mobility handover process in the network layer is tackled by a trigger developed AMH message to the next access point. Thus, a mobile node keeps communicating with the current access point while the network layer handover is executed by the next access point. The mathematical analyses and simulation results show that the proposed scheme performs better as compared with the existing approaches.
\end{abstract}

\section{Introduction}

Wireless networks are loomed as a promising technology that can provide seamless access to network services. The past decade has witnessed a rapid increase of network services in the Internet at any time and any place. In mobile IP networks, this sharp increase of network services requires seamless mobility and low delay during mobile node's (MN) movement. This can be achieved by a seamless mobility handover management scheme with low signalling cost. Moreover, the quality-of-service (QoS) of ongoing network applications is glaringly affected by mobility handover processes, due to high delay and packet loss. Besides, the support of an efficient mobility management is considered as a one of the very important issues for the future generation of wireless and mobile networks services [1-3].
Basically, the handover signalling process which is used in obtaining the new IPv6 address from visited network (network layer handover) must wait until the link layer handover has performed its own processes. Accordingly, the initiation time is increased and afterward, the overall handover latency is increased similarly. This can be considered as a main reason of degrading the QoS in wireless local area networks (WLANs) $[4,5]$. Therefore, the efficient handover process is challenging as a way to achieve a seamless mobility with low handover delay [5-8].

In order to keep the routability between MN and access routers (AR), the IETF has designed Mobile Internet Protocol version 6 (MIPv6) to provide mobility in mobile devices. Within MIPv6 The MN expected to be supplied with IPv6 address whether it is currently connected to the AR belong to its home agent (HA) or other networks with different ARs and 
configured with different global routing prefixes. Therefore, the MN must obtain a temporary new IPv6 address instead of its home address (an IPv6 address assigned by HA of MN which is started with the home subnet prefix) of HA. During the time $\mathrm{MN}$ is connected to its HA, all the traffic of packets are addressed to its configured home address. Afterwards, all the packets that were sent by CNs are routed to the MN's HA employing the typical Internet routing mechanisms [9].

On the other hand, when an $\mathrm{MN}$ is connected to some foreign networks, it utilizes one or more of care-of-addresses $(\mathrm{CoA})$, that is, depending on the number of visited foreign networks. The assigned CoA is an IPv6 address with a subnet prefix indicating the visited foreign network. By the time $\mathrm{MN}$ is active with a particular foreign network, the packets are addressed with the obtained CoA to be reachable to the $\mathrm{MN}$. Moreover, in some cases, $\mathrm{MN}$ can accept packets from a number of CoAs, for instance, when it is roaming but still accessible at the previous network.

In MIPv6, there are some issues that still need to be addressed regarding mobility handover process. When $\mathrm{MN}$ performs a fast movement, the signal quality of the current access point (AP) can go down rapidly. Thus, $\mathrm{MN}$ is not able to connect for a long period with the current AR (CAR), for instance, when MN does not have sufficient time to send handover signals which are responsible for new IP address registration process. Thus, it can lead to mobility handover failure. In other words, the mobility handover process, which is performed by $\mathrm{MN}$ to obtain a new CoA from visited network, in addition to the registration processes with $\mathrm{HA}$ is negatively affected by inefficient mobility handover process. Consequently it can degrade the QoS of ongoing applications due to high overall handover delay.

In the light of discussions given above, the handover delay is one of the hot issues that are normally experienced within MIPv6 and other well known mobility management protocols [5]. Therefore, it is important to take into consideration this aspect as a way to improve the network layer handover processes. Moreover, the handover delay of several seconds is unacceptable for most of time-sensitive and lost-sensitive applications (real-time applications) [10]. Thus, many mobility handover schemes have been proposed. In this regards, several approaches have been proposed as a way to reduce the handover latency that is experienced within MIPv6 due to IPv6 address configuration and registration processes.

The remainder of the paper is as follows. Section 2 provides the related literature in mobility handover process of network layer and mobility management approaches, and Section 3 presents the detailed design of proposed $\mathrm{AMH}$ scheme, whereas Section 4 illustrates the performance evaluation of proposed AMH scheme followed by the conclusion in Section 5.

\section{Related Works}

One of the essential issues in wireless communications is the handover delay. Hence, many studies have tried to overcome with appropriate solution to decrease the associated time delay during handover process. In order to maintain the mobility handover process of network layer, firstly the Internet Engineering Task Force (IETF) has proposed various IP-based mobility management schemes, such as MIPv6, Hierarchical Mobile IPv6 (HMIPv6), and Fast MIPv6 (FMIPv6) [11-14]. The goal of these schemes is to maintain the communication continuity when an MN moves between different ARs $[15,16]$. However, these mobility protocols still have shortcomings in terms of handover latency, packet loss, and signaling overheads [16].

A hierarchical mobile IPv6 (HMIPv6) is proposed by Castelluccia [17] as a way to mitigate the signaling overhead that is caused by the binding update processes. This was achieved by offering some kind of local HA, called mobility anchor point (MAP), in addition to complaining subnetworks into clusters called MAP domains, where each cluster is controlled by a single MAP. However, there are still some issues visible in HMIPv6, for instance, when any single of MAP is failed leads to loss all the communication. Moreover, the performance of real-time applications is not considered in the design phase of HMIPv6 [18].

Furthermore, within HMIPv6, there is a lack of inclusive analysis of different parameters in terms of signaling load. In addition, there is an ignorance of binding refresh cost that is performed after the binding lifetime is expired. On the other hand, the impact of packet tunnelling cost, which could be high due to the high mobility handover delay, was experienced by MN. Thus, many packets could be waiting to be forwarded to the MN after the routability is returned.

As mentioned before, within MIPv6 and HMIPv6 processes there are still some problems such as handover latency, packet loss, signaling overheads, and extensive MIPv6 functionality in the IPv6 load in MN side [19]. Therefore, FMIPv6 [20] has been proposed by IETF as an extension to support the mobility management and improve the performance of MIPv6. This new extension relies on some of the link layer triggers in order to execute network layer handover processes in a fast way [21]. This process is performed by preparing the new IP configuration before the current link breaks down. This was achieved by enhancing the employed ARs as a way to perform a fast binding update (BU) message between previous access router (PAR) and new access router (NAR). Throughout this process the $\mathrm{MN}$ was able to send and receive packets once it is attached to the new network link. FMIPv6 assumes that the new CoA (NCoA) configuration messages are received through the current network (the PAR) before the link layer handover is began. This was handled by sending some of network layer signals (which related to the new IP address registration process) during the link layer handover processes to the NAR.

However, FMIPv6 protocol mainly relies on two modes that are reactive and proactive modes, whereby at best the employed anticipation method by proactive mode does not guarantee reliable packet transmission [18]. Moreover, the unpredictable and fast movements that are performed by $\mathrm{MN}$ can cause packet loss.

In contrast, a network based mobility management protocol proxy MIPv6 (PMIPv6) [22] was developed as a way to reduce the signaling overhead of MIPv6. Two new elements 
were introduced by PMIPv6, mobility access gateway (MAG) and local mobility anchor (LMA). The updating process of MN's movement is identified by the MAG, which is a function located at the AR. Thus, MAG updates to the LMA which is to perform the functions of $\mathrm{HA}$ in addition to managing the routing process of data packet to the MN. Subsequently, the $\mathrm{MN}$ was able to change its locations without any signaling generated by MN's side. When the MN is registered at the LMA, the same home network prefix (HNP) is provided by LMA to the MN. The HNP is sent by employing a proxy binding acknowledgement message, which responds to a proxy binding update message sent from the MAG.

However, PMIPv6 was developed mainly for the local mobility management, which does not support a global mobility management $[15,23,24]$. For instance, when some MNs support only MIPv6 and intend to use PMIPv6, the network infrastructure should provide MIPv6 functions to them. Therefore, the MAG in this case cannot act like the HA. The MAG is supposed to advertise its own routing prefix instead of the IPv6 prefix that the MN always receives when connected to any AP within PMIPv6 domain (MN-HoA). Accordingly, these MNs without implementation of PMIPv6 need to rely on the network to manage their mobility. Hence, it can be noticed that the PMIPv6 is facing scalability issues when MNs from global network prefixes enter its network domains.

Although, some approaches recently are tried to enhance the functionality of PMIPv6, until now they could not efficiently support the seamless mobility handover $[15,16]$. The reason is that within PMIPv6 a high handover delay is normally experienced by intersubnet handover. Thus, high packet loss could occurr especially with real-time applications due to the high packet rate. Moreover, in case of any single failure of a MAG can lead to degrade the performance to be worst than standard MIPv6.

In order to cope with the issues that normally occurred by adding the authentication, authorization, and accounting (AAA) functions with the MIPv6 handover processes, such as the high cost delay of authentication and registration process, the authors in [25] developed a novel hierarchical authentication scheme. The proposed method focused on minimizing the authentication latency in AAA processing. In the proposed scheme, a hierarchical AAA architecture was achieved, in which the AAA servers are deployed on the MAP. The authors analyzed the signaling cost of the mobility handover process of the proposed authentication registration (AuthR) method.

AuthR method is considered the total cost of mobility handover process, $C_{\text {total }}$, which contains three parts, the transmission cost of registration signaling $\left(C_{\text {reg }}\right)$, the transmission cost of authentication signaling $\left(C_{\text {auth }}\right)$, and packet delivery cost $\left(C_{\text {trans }}\right)$. The costs of registration and authentication signaling are considered together in the proposed AuthR method, which is called $C_{\mathrm{RA}}$. Furthermore, $C_{\text {trans }}$ was indicating the packet processing and delivery cost during mobility handover process. Total cost of mobility handover process was given by

$$
C_{\text {total }}=C_{\text {reg }}+C_{\text {auth }}+C_{\text {trans }}=C_{\mathrm{RA}}+C_{\text {trans }} \text {. }
$$

However, it can be observed from Formula (1) that the authors did not consider the signalling delay and overhead of registration and address configuration processes. In other words, the registration and address configuration signaling overheads can contribute in increasing the total handover delay. On the other hand, due to security issues that could be araised when MNs perform many changes of their positions throughout the IPv6 network while retaining their existing connections these processes need to support low delay time [26], many advantages are thus attained, but obtaining low signalling overhead or cost with considering security aspects remains a fundamental concern [27].

An improved fast handover scheme for hierarchical mobile IPv6 (IF-HMIPv6) was proposed in [28], in order to reduce the handover delay and packet loss. By the time the $\mathrm{MN}$ sent an improved router solicitation for proxy (IRtSolPr) message to the MAP, the procedures of IF-HMIP method began during the link layer handover process. The IRtSolPr message contains information about the MAC address or identifier of the AP. Moreover, as a way to identify the MAP that MN follows during IF-HMIPv6 processes, an "I flag" in IRtSolPr message was included. By using this "I flag," MAP was able to process the new CoA configuration process on behalf of the MN and sent the handover initiation (HI) message to the NAR in order to establish a tunnel.

Consequently, the MAP sent the proxy router advertisement (PrRtAdv) message to the $\mathrm{MN}$ as a response to the "I flag," which was sent via IRtSolPr message of MN as an indicator for handover initiation processes. Thus, $\mathrm{MN}$ is waiting for the FBAck instead of sending the F-BU to the MAP before it received the F-BAck. The duplicated address detection (DAD) process was processed by NAR to verify the new CoA. DAD process was performed after NAR is received and the HI sent by MN. When DAD process is verified by the new CoA, a response with a handover acknowledgement message (HAck) was sent by AR to MN. Eventually, the tunnel is established between MAP and NAR.

Furthermore, an evaluation based on numerical model of proposed IF-HMIPv6 was conducted during the study. Formula (2) shows that the time delay within one MAP is represented by L2's delay only, whereas Formula (3) illustrates the time delay that occurred during handover processes between different MAPs, where $T_{\mathrm{BU}(M-\mathrm{HA})}$ is the time, is required to perform binding update to HA by local MAP. Consider the following:

$$
\begin{gathered}
T_{\text {IF-HMIP-inter }}=T_{L 2}, \\
T_{\text {IF-HMIP-inter }}=T_{L 2}+T_{\text {detect }}+T_{\mathrm{CoA}}+T_{\mathrm{BU}(M-\mathrm{HA})} .
\end{gathered}
$$

However, it can be observed from Formula (3) that the time was consumed in address configuration process of $\mathrm{CoA}$ inside one local MAP that is still visible. Moreover, this study did not discuss the consequences behind increasing the number of MNs. In other words, the negative effects which lead to signaling overhead then decrease the QoS on going applications due to high number of MNs.

On the other hand, a seamless flow mobility management architecture (SFMMA) for vehicular communication networks was proposed in [29]. The proposed SFMMA method 
was introduced as a way to cope with the mobility management aspects in vehicular ad hoc networks (VANETs), in order to maintain the wireless connection with roadside network service nodes during vehicular movement. The authors in [29] are designed a multiaccess wireless network architecture employing the Wi-Fi (WLANs), vehicular, worldwide interoperability for microwave access (WiMAX), and long-term evolution 4G (LTE) technologies, supporting a continuous and wireless connection for the ongoing vehicular applications. This method was devised for the needs of each application class, such as throughput, delay, and packet loss. The status of the current active wireless connection was considered in order to tackle the division of the mobility flow. Furthermore, the authors elaborated the handover decision using three elements that are based on proxy MIPv6 (PMIPv6), the local mobility anchor (LMA), the mobility access gateway (MAG), and MN.

However, at best, by using the SFMMA scheme MN still asking information from the MAG where the network interface is connected; inquires about network status and the home network prefixes (HNP). Throughout these amounts of information, MN can indicate whether the interface is still active or not; otherwise it does not know to whom it needs to route the packets and what are the prefixes that can be used to send its packets. Furthermore, the triggering process of mobility handover decision can be performed by many sides as it was stated in the proposed network architecture, which are MN, LMA, and MAG. These kinds of decisions can lead MN to experience unnecessary handovers [30]. The reason is that when a rapid changing occurrs with MN's position due to roaming process, many mobility aspects are changeable that in such circumstances (high speed) it takes time in updating the parameters with both LMA and MAGs. Therefore, it could be more difficult to include all MNs, LMAs, and MAGs in mobility handover decision making processes that lead to unstable handover decision. Besides, this can result in an increase in the signaling costs and overheads of the entire handover processes.

A host-based localized mobility management (LMM) scheme was proposed by Cho et al. [5]. The developed scheme supported multiple care of addresses and fast handover mechanism. The handover processes within LMM scheme are controlled using a developed localized mobility client (LMC) server. The connection was established by creating a tunnel by LMC as a way to process the location registration. A BU message was sent using tunnel in addition to handle the $\mathrm{MN}$ communication over this tunnel. After the link is established with candidate network, the CoA is formed to be assigned for $\mathrm{MN}$.

However, the proposed scheme still offers complexity in network handover signaling process. This due to the fact that introducing localized mobility management clients, servers, and Tunnel Gateways (TG); with any failure of one of them it degrades the whole system performance. Moreover, it can be noted that the MN still engages with many of control signals as it was demonstrated in [5]. In other words, the tunnel establishment trigger and the BU messages are still visible within MN's side, which keeps the overheads over MN during mobility handover processes. Furthermore, MNs still inquire CoA from visited networks in order to obtain the IP connectivity, which also requires more time delay.

\section{Advanced Mobility Handover Scheme (AMH)}

In this section the detailed design of the AMH scheme is discussed by illustrating perspective architecture of AMH to explain how the handover process is performed. After the handover decision with one particular AP is taken, the AMH scheme will be triggered by $\mathrm{MN}$. The triggering message is enclosed in an association request frame during the IEEE $802.11 / \mathrm{b}$ handover process. The $\mathrm{AMH}$ process is triggered as a way to inform the $\mathrm{HA}$ and $\mathrm{CN}$ the changes in the network layer (the changes with associated AR) of the wireless networks through the new selected AP. In AMH architecture there are ARs connected to the Internet service provider (ISP) which relies on IPv6, APs, and MN being mobile across the deployed APs which is illustrated in Figure 1. These APs can access the Internet connection through ARs. Figure 1 shows the mobility scenario when MN crosses different APs and different ARs. The internal configured subnet addresses for AR1, AR2, and AR3 are (2001:1:1:1:/64), (2001:1:2:1:/64), and (2002:1:1:1:/64), respectively. These addresses are identifying three different subnets, whereby the global routing link is configured with the same routing prefixes for both AR1 and AR2, whereas AR3 with different routing prefix of (2002:1:1:2:/64). Thus, the main difference between AHM2 and AMH3 is that, when an MN moving from AP2 to AP3, the subnet IPv6 address within connected AR is changed as well as the APs IPv6 address, but the global routing prefix of global connection is still the same for both AR1 and AR2; this is during $\mathrm{AMH} 2$ process. On the other hand, in $\mathrm{AMH} 3$ both IPv6 addresses of subnet of AR and global routing connection are different. Hence the presented processes by AMH3 design will be implemented to tackle this scenario.

During the roaming process the $\mathrm{MN}$ uses a unique home IPv6 address assigned by HA in order to keep traffic flowing without a CoA. Therefore, the proposed AMH scheme introduces a unique IPv6 address which can be used by the $\mathrm{MN}$ during the roaming process. The introduced unique IPv6 address was based on standard 128-bit IPv6, which can be used by the $\mathrm{MN}$ during the roaming process for global mobility purposes. It is worth mentioning that the proposed unique local IPv6 unicast addresses by [31] could not achieve the globality in maintaining the network connection using the obtained unique IPv6 addresses [32].

These unique IPv6 addresses in AMH scheme were achieved by allocating three fields which are global routing prefix field (with high probability of uniqueness), AP-ID, and MN-ID fields in the IPv6 address of any MN, AR, and AP. The IPv6 addresses have been configured in each AP by first assigning the global routing prefix, which is a field of the IPv6 address indicating the APs that are connected to the same AR. The second developed field of the AP IPv6 address is the APID which uniquely identifies each AP and the third developed 


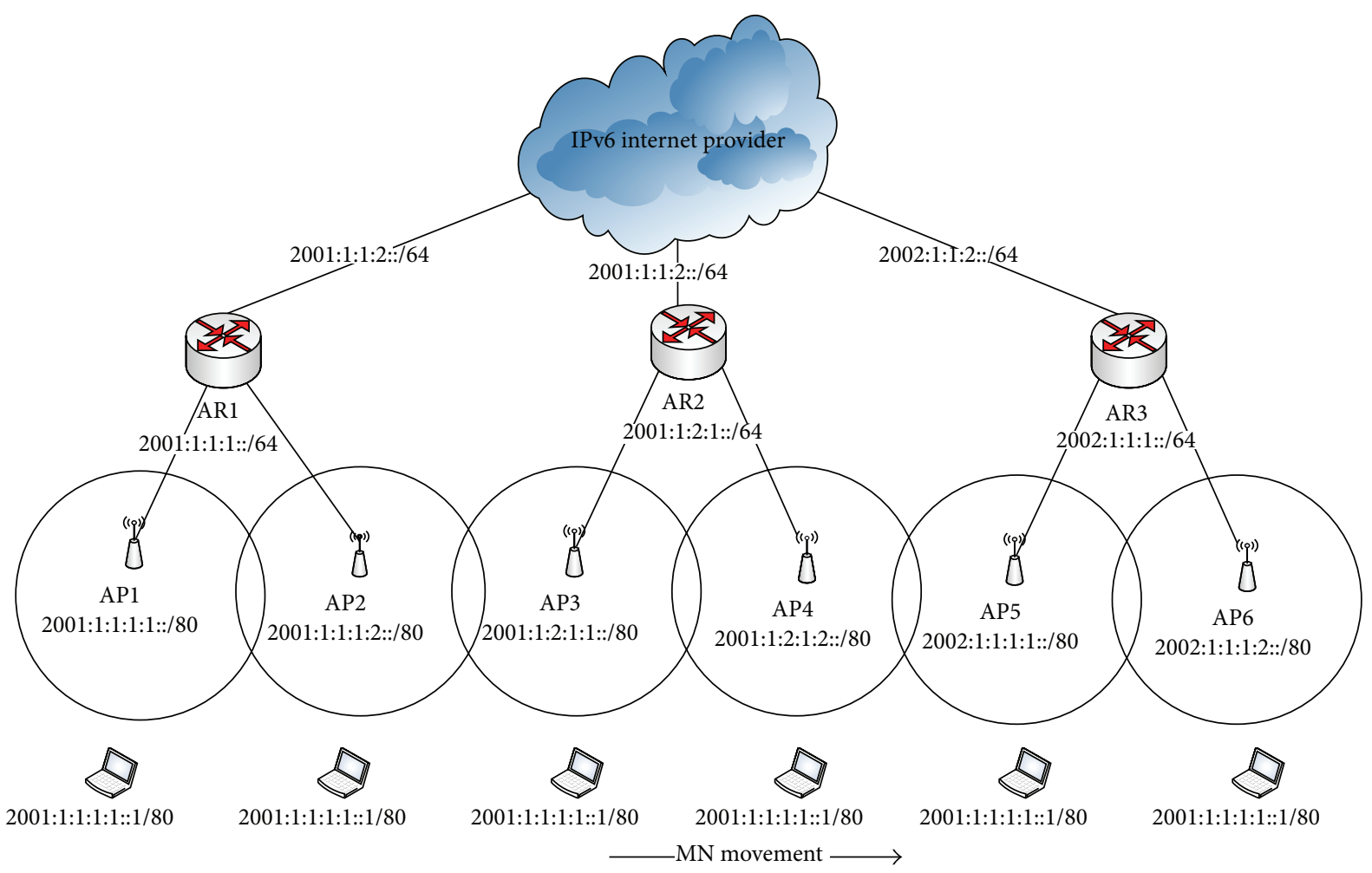

Figure 1: Proposed MIPv6 addressing scenario for AMH.

field of the IPv6 address is the MN-ID in AP, which is assigned to zero in the IPv6 addresses of APs and ARs. On the other hand, the IPv6 address for each MN during the first round of $\mathrm{AMH}$ process is uniquely identified by $\mathrm{HA}$ using the MN-ID field. Moreover, another temporary $\mathrm{MN}-\mathrm{ID}_{\text {temp }}$ is generated by $\mathrm{AP}$ in each time an $\mathrm{MN}$ was associated with that particular AP and temporarily saved in a developed table inside the AP.

The first step of configuring the proposed unique IPv6 is creating the global routing prefix field. The bit length that utilized to configure this field was assumed to be 64 bits, because the availability of high utilization ratio does not require internal structure based on [31]. Therefore, this allocation was used to achieve high probability of uniqueness and avoid any clash situation with other assigned IPv6 addresses to an MN in the structured wireless network. The pseudorandom method [33] was employed in allocating the ID number of global routing prefix field during each time the unique IPv6 address is generated. Apart from this method, the way of generating the ID number of a particular global routing prefix is not sequential but is randomly identified with high probability of uniqueness. Similarly, the same pseudorandom method was used in obtaining the unique MN-IDs and MN-ID temp, which afterwards were utilized in global routing purposes when the MNs visiting an AR configured with IPv6 with different global routing prefix (more explanation about this process was discussed by AMH3 processes in Section 3.3). On the other hand, the AP-ID of the deployed APs in simulated scenario was configured uniquely as a way to avoid the conflicts that might occurred among them. AP-ID field was set to $i$ equals to 16 bits in the proposed IPv6 format.
The probability of proposed unique IPv6 address to be clashed with other assigned MN's unique IPv6 addresses was given by

$$
P_{\text {unique IPv6 collision }}=1-\exp \left(\frac{-\mathrm{MN}_{\text {number }}^{2}}{2^{\left(\mathrm{GRP}_{L}\right)}}\right),
$$

where $\mathrm{MN}_{\text {number }}$ is the number of MNs that assigned with unique IPv6 addresses and $\mathrm{GRP}_{L}$ is the bit length of global routing prefix field. Thus, for instance, when the number of associated MNs is 10000 and the $\mathrm{GRP}_{L}$ is 64-bits; the $P_{\text {unique IPv6 collision }}$ equals $2.71 \times 10^{-12}$ which infers a very low probability of collision.

Figure 2 shows the IPv6 address format and data structure in table form saved in each HA, AR, and AP in the proposed AMH scheme. Three IPv6 fields (IPv6 address format) are illustrated in Figure 2 where $i$ is set to 16 bits. Moreover, the data structure in table form is shown in Figure 2. This table is designed and integrated into the proposed $\mathrm{AMH}$ in order to ensure that the IPv6 address of AP and AR during the MN roaming process is updated. These tables are utilized in AP, $A R$, and HA. Figure 2 shows the data structure in table form, known as (local MN table), which has been saved in HA. This local MN table in HA contains the MN's IPv6 address along with the IPv6 address of the AR that the MN is currently connected as shown in Figure 2. The routing table in each AR ( $\mathrm{MN}$ routing table) consists of the IPv6 address of the MN with MN's ID in case the MN is attached to an AR with a different global routing prefix and AP's IPv6 address where the $\mathrm{MN}$ is currently associated with as shown in Figure 2. 
TABLE 1: Content of modified association request frame send by MN and AMH procedure process by next AP.

\begin{tabular}{|c|c|}
\hline Body information & $\mathrm{AMH}$ \\
\hline Default information + MN IPv6 address $+(01)$ & Reserve memory space, establish MN-ID temp, and execute AMH1 \\
\hline Default information + MN IPv6 address + (10) & Reserve memory space, establish $\mathrm{MN}$ - $\mathrm{ID}_{\text {temp }}$, and execute $\mathrm{AMH} 2$ \\
\hline Default information + MN IPv6 address + (11) & Reserve memory space, establish MN-ID temp , and execute AMH3 \\
\hline
\end{tabular}
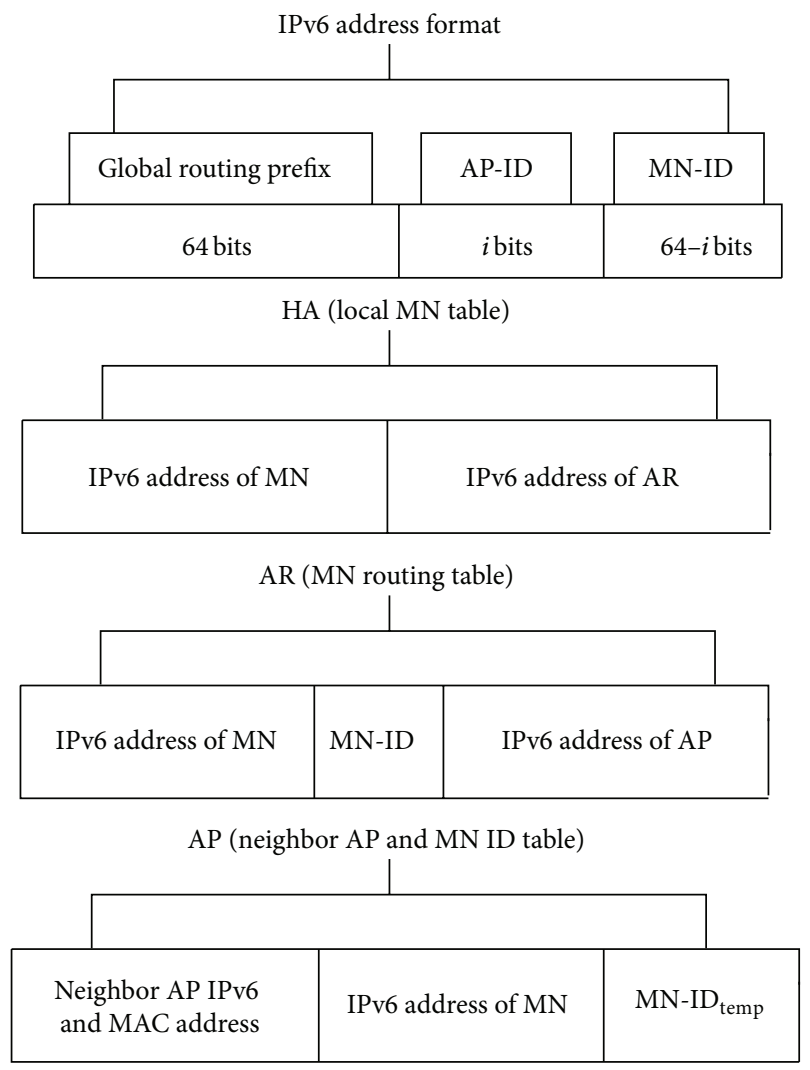

FIgURE 2: The format of the proposed unique IPv6 address and data structure as a table form saving in each of HA, AR, and AP in the proposed AMH scheme.

Finally, in each particular AP the table of the neighbor of AP's IPv6 and MAC address, current associated MNs IPv6 addresses, and MN's ID for each MN created by AP after the association process are saved as a table as shown in Figure 2.

In order for the $\mathrm{MN}$ to trigger the $\mathrm{AMH}$ scheme in the time the handover decision has been taken, the $\mathrm{AMH}$ message has been developed. In standard 802.11/b there are various frame types defined to be used by $\mathrm{MN}$ and $\mathrm{AP}$ for communications, as well as managing and controlling the wireless link. The association request frame in Standard $802.11 / \mathrm{b}$ is sent by $\mathrm{MN}$ to AP in order to allocate resources for the $\mathrm{MN}$ and to synchronize with a radio network interface card (NIC). The association request frame is a management frame as defined in [34]. As illustrated in Figure 3(a), DA is the destination address, SA source address, and BSSID basic service set ID which is equivalent to AP ID and FCS frame check sequence. Basically, the frame body of a management frame of subtype association request contains information about the MN such as supported data rates, the SSID of the network the MN intends to associate with, and some other information. In the proposed AMH scheme the association request frame's body is modified by adding 130 bits. The IPv6 address of the $\mathrm{MN}$ uses 128 bits in each association frame plus 2 bits for the $\mathrm{AMH}$ triggering the $\mathrm{AMH}$ message.

In the proposed AMH architecture three handover cases are considered which are handovers within the same subnet $(\mathrm{AMH1})$, with different subnets and same global routing prefix $(\mathrm{AMH} 2)$ and with different subnets and different global routing prefix (AMH3). Figure 3(b) depicts the modifications that have been done on the association request frame that the MN sends to AP. The IPv6 address of the MN is included in the body of association request frame each time a request is sent to the new selected AP. Moreover, the AMH message code included in each association request frame is sent by the $\mathrm{MN}$ in order to trigger the AMH scheme by the selected AP. Table 1 shows the three types of association request frame sent by the MN and the AMH procedure which is executed by the next AP. When the MN includes the AMH code (01) a request is made of the selected AP to reserve memory space and establish the MN-ID temp which will later be used as a route distinguisher of the packets which are directed to the

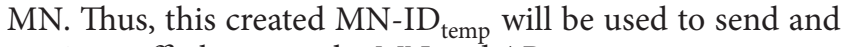
receive traffic between the $\mathrm{MN}$ and $\mathrm{AP}$.

Moreover, when the AP receives the AMH code (01) the $\mathrm{AMH} 1$ procedure will be triggered in order to perform the mobility handover on behalf of the MN. In addition, when $\mathrm{AP}$ receives an association request frame from the $\mathrm{MN}$, it includes AMH code (10), which means repeating the previous procedure with $\mathrm{AMH} 2$ process. At the same time if the received $\mathrm{AMH}$ code sent by $\mathrm{MN}$ is (11), AP will handle the AMH3 procedure. This AMH message code is placed in the two modified bits in the association request frame as illustrated in Figure 3(b). These three different AMH handover procedures $\mathrm{AMH1}, \mathrm{AMH} 2, \mathrm{AMH} 3$ are discussed in detail by the following sections with practical examples of table entries updated in AP, AR, HA, and CN.

3.1. Advanced Mobility Handover Scheme within the Same Subnet (AMH1). Two APs are considered to be in the same subnet when both of them are connected to the same AR (that belong to one subnet). In this scenario, when the $\mathrm{MN}$ moves from current AP to the next, the RSS is gradually decreasing with the current AP in which communication is to continue. At this point in time, which is called passive scanning, the $\mathrm{MN}$ receives beacon frames from the next $\mathrm{AP}$ in the vicinity. Basically, beacon frames are periodically transmitted by the AP to announce the presence of a WLAN network. Through these beacon frames, MN can extract the next AP's IPv6 address using the obtained new AP MAC 


\begin{aligned} & Octets: 2 \\
& $\mid$\begin{tabular}{|c|c|c|c|c|c|c|c|}
\hline $\begin{array}{c}\text { Frame } \\
\text { control }\end{array}$ & Duration & $\begin{array}{c}\text { Address } \\
1 \text { (DA) }\end{array}$ & SA & BSSID & $\begin{array}{c}\text { Sequence } \\
\text { control }\end{array}$ & $\begin{array}{c}\text { Frame } \\
\text { body }\end{array}$ & FCS \\
\hline
\end{tabular} \\
& \hline\end{aligned}

(a) Standard management frame format

\begin{tabular}{l} 
Octets: 2 \\
\begin{tabular}{|l|c|c|c|c|c|c|c|c|}
\hline $\begin{array}{l}\text { Frame } \\
\text { control }\end{array}$ & Duration & $\begin{array}{c}\text { Address } \\
1(\mathrm{DA})\end{array}$ & SA & BSSID & $\begin{array}{c}\text { Sequence } \\
\text { control }\end{array}$ & $\begin{array}{c}\text { MN } \\
\text { IPv6 } \\
\text { address }\end{array}$ & $\begin{array}{c}\text { AMH } \\
\text { message }\end{array}$ & FCS \\
\hline
\end{tabular} \\
\hline
\end{tabular}

(b) The modified association request frame

FIgURE 3: The management frame format and modified association request frame.

TABLE 2: Data updating in each of AP and AR after AMH1 message.

AP2 entry table after T2

AR1 entry routing table after T2

2001:1:1:1:1::1/80 + MN-ID temp $(1)$ 2001:1:1:1:1::1/80 + 2001:1:1:1:2::/80

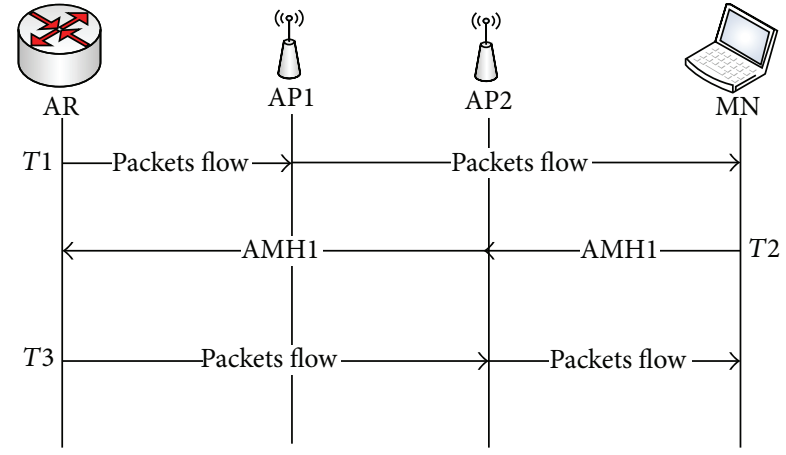

FIGURE 4: AMH1 scheme within the same subnet.

address by searching for it in the created neighbor AP's IPv6 list of current APs, which is presented by the table structure of AP in Figure 2. Utilizing this process, the MN realizes whether the next AP belongs to the same current subnet or not. This process is performed during time point $T 1$ as shown in Figure 4. Thus, using this obtained IP address the MN can determine if any changes have occurred with the subnet using AP-ID which is uniquely identified by each AR for associated APs as mentioned before. In case the next AP is attached to the same subnet, the $\mathrm{MN}$ sends the AMH1 message code included in the association request frame to the next AP (AP2) as shown in Figure 4, asking that the AMH1 handover process be triggered during period $T 2$.

Figure 4, illustrates the execution procedure of AMH1 scheme within the same subnet. As depicted in the figure, the MN sends the AMH1 a message which contains its IPv6 address along with code for AMH1 using the association request frame to the AP2. When the next AP (AP2) receives this frame it performs the default process of association request in addition to establishing the new $M N-\mathrm{ID}_{\text {temp }}$ that will be used by AP in packet forwarding processes.
The $\mathrm{MN}-\mathrm{ID}_{\text {temp }}$ establishment process was done by $\mathrm{AP}$, utilizing the reserved 16 bits within the developed unique IPv6 address which is allocated on MN-ID's field. When an AP accepted MN's association request, it creates an ID which is represented by a decimal number to uniquely identify the $\mathrm{MN}$, which is placed on the 16 bits of MN-ID field. AP then forwards AMH1 message to the AR. This AMH1 message sent by AP2 contains the IPv6 address of the MN and IPv6 address of the AP2. After AR receives the AMH1 message coming from $\mathrm{AP} 2$, it checks its $\mathrm{MN}$ routing table and updates the IPv6 address to be the IPv6 address of AP2 in the routing entry. It can be observed that during the time consumed by the AMH1 scheme, the MN kept the connection to AP1 in the link layer. Therefore, it could receive the data forwarded by AP1. Thus, the MN is still able to receive the data destined and forwarded to the home IPv6 address even though the link attachment changed. Afterward, when AMH1 has been completed, the MN disconnected from the AP1 in the link layer and started to receive data from the AP2 in time T3. Thus, the AMH1 ensures packet flow between the MN and AR during the communication as shown in Figure 4.

Table 2 shows the data updating in AP2 and AR1 which are illustrated in Figure 1 as a practical example for AMH1. This presented example shows that when the MN moves from AP1 towards AP2 both are associated to AR1. The IPv6 address of the MN has been added in AP with MN's ID created by AP2 which is (1) in this present example. After $\mathrm{AMH} 1$ is received by AR1, the routing table is updated by replacing the IPv6 address of AP1 with the obtained IPv6 address of AP2, to be placed as the new active AP for MN with IPv6 address of (2001:1:1:1:1::1/80). Thus, when the packet traffic was sent to AR1 forward it to the new AP's IPv6 address of AP2.

3.2. Advanced Mobility Handover Scheme within Different Subnets and the Same Global Routing Prefix (AMH2). As mentioned earlier the $\mathrm{MN}$ can extract all information related 

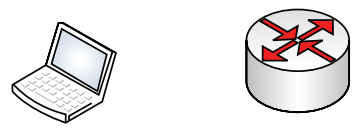

$\mathrm{CN}$

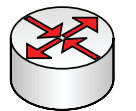

AR2

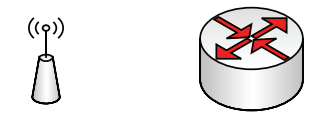

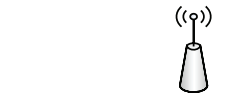

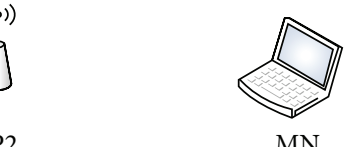

AR1

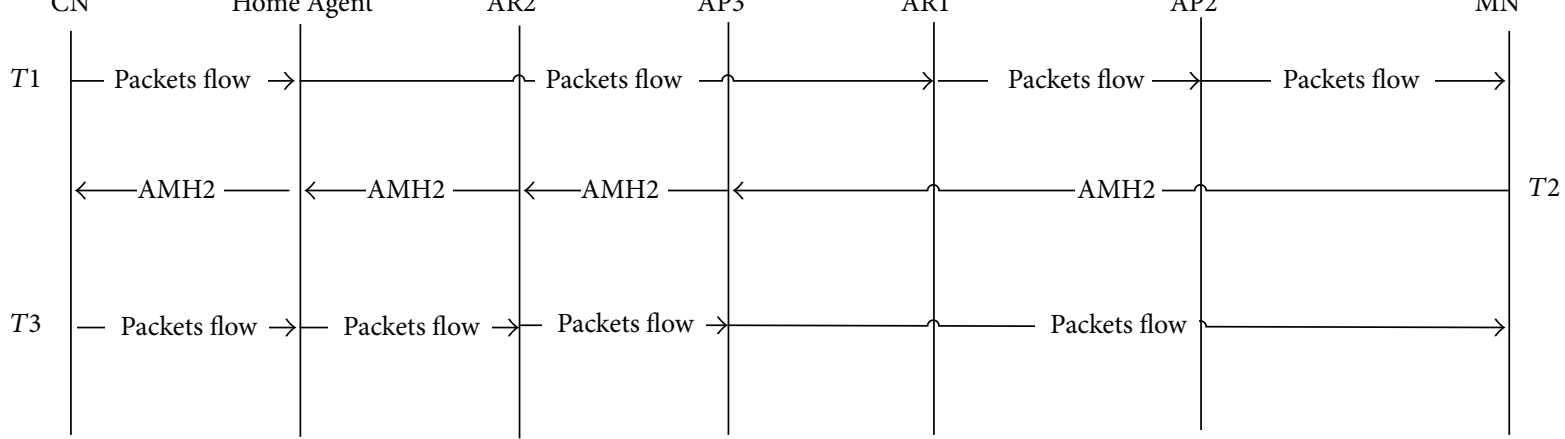

FIGURE 5: AMH2 scheme within different subnets and the same global routing prefix.

TABLE 3: Data updating in each of AP, AR, HA, and CN after AMH2 message.

\begin{tabular}{lcc}
\hline AP3 entry table after T2 & AR2 entry routing table after T2 & HA\&CN entry routing table after T2 \\
\hline 2001:1:1:1:1::1/80 + MN-ID temp $(1)$ & 2001:1:1:1:1::1/80 + 2001:1:2:1:1::/80 & $2001: 1: 1: 1: 1: 1 / 80+2001: 1: 2: 1:: / 64$ \\
\hline
\end{tabular}

to the next AP through its beacon frame during time point $T 1$. When the MN realizes that the next AP belongs to a different subnet but the global routing prefix is the same as the current $\mathrm{AP}$, the AMH2 scheme for different subnets and the same global routing prefix will be triggered in T2. Figure 5 shows that the MN sends the AMH2 message to the next AP (AP3) to trigger $\mathrm{AMH} 2$ while the $\mathrm{MN}$ is still communicating with the current AP (AP2). When AP3 receives the AMH2 message sent by the $\mathrm{MN}$, the AP3 creates the $\mathrm{MN}-\mathrm{ID}_{\text {temp }}$ and then forwards AMH2 to the associated AR2 which contains the MN and AP3 IPv6 address. Once the AMH2 message reaches $A R 2$, the routing table will be updated by adding the IPv6 address of AP3 and that of the MN which it is intending to communicate with. The AMH2 message will then be forwarded via AR2 to the HA of the MN through the Internet and the payload of this message will include the IPv6 address of the MN and the IPv6 address of AR2.

After receiving $\mathrm{AMH} 2$ the $\mathrm{HA}$ then updates the IPv6 address of the associated AR to the address of AR2 and forwards the AMH2 message to the $\mathrm{CN}$ which contains the IPv6 address of the MN and AR2. Moreover, AR2 established the tunnel between itself and AR1 as a way to forward all the cached packets' flow that was visible in T3. It is worth mentioning that the tunnel establishment order that was triggered by NAR is based on the code presented within AMH2 message (10). The reason of this tunnel is to ensure reroute and old packets that were destined to the previous AR before mobility handover was completed with NAR. Thus, those buffered packets will be delivered to MN via NAR after it was physically connected to NAR. Eventually, when CN received the AMH2 message it updated its routing entry with the new IPv6 address of AR2 in order to retain the routability with the MN through the newly visited AR2 with a different subnet but the same global routing prefix. Figure 5 also shows that during the time of $\mathrm{AMH} 2$ processes, the $\mathrm{MN}$ was still connected to AP2 in the link layer. Therefore, the packet flow forwarded by $\mathrm{CN}$ and HA through AP2 can still be received and data continuity has been ensured using AMH2. Figure 1 illustrates the presented $\mathrm{AMH} 2$ process scenario when the $\mathrm{MN}$ was moved from AP2 that belongs to AR1 toward AP3 that is associated with AR2.

Table 3, shows the practical example of data updating in $\mathrm{AP}, \mathrm{AR}, \mathrm{HA}$, and $\mathrm{CN}$ after $\mathrm{AMH} 2$ message was triggered by the MN. It can be observed that the AP's IPv6 address in AR2 is updated with AP3 address (2001:1:2:1:1::/80) in which the MN will use to communicate with during time $T 3$. Afterwards, the routing tables of $\mathrm{HA}$ and $\mathrm{CN}$ are updated by a new AR entry, (IPv6 address of AR3) which it obtained after sending AMH2 message from AR3 to HA and $\mathrm{CN}$ through the internet. Thus, all the packets that are forwarded to MN will be redirected to the new visited AR3 during T3 using the established tunnel between AR2 and AR1. In other words, the IPv6 address was updated in AR, HA, and CN after the $\mathrm{MN}$ triggered an AMH2 message with code (10) to AP3 during time T2 while still connected to AP2 that belongs to AR1, as can be seen in Figure 5. Subsequently, the advanced mobility handover was tackled via AP3 on behalf of the MN which allows the $\mathrm{MN}$ to save the time normally consumed for this process to be performed prior to its default time (after link layer handover is completed). Hence, MN through the proposed AMH scheme could achieve an efficient IPv6 address configuration and registration process.

3.3. Advanced Mobility Handover Scheme within Different Subnets and Different Global Routing Prefixes (AMH3). $\mathrm{AMH} 3$ has been developed in order to use the AMH scheme to tackle the handover when the $\mathrm{MN}$ visits a new network with a different global routing prefix. More precisely, when the visited networks are configured with a different global routing prefix the $\mathrm{MN}$ sends $\mathrm{AMH} 3$ message to the newly 


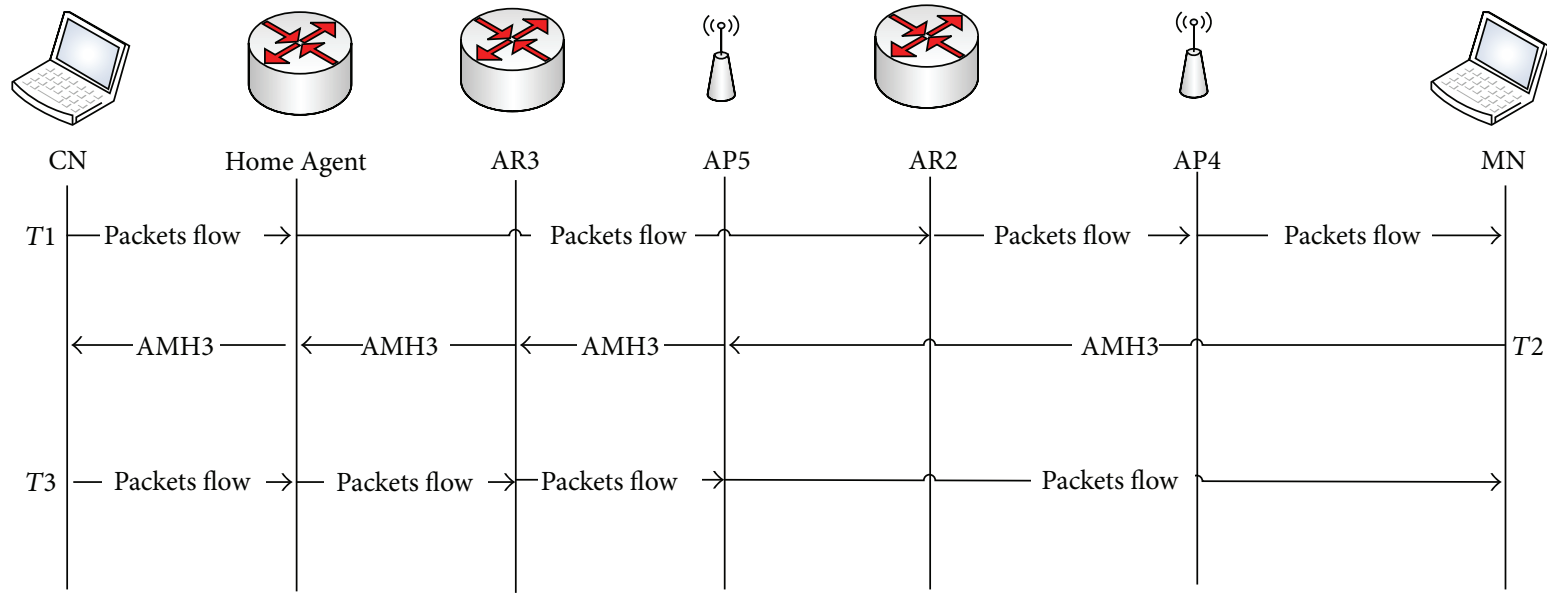

FIGURE 6: AMH3 scheme within different subnets and different global routing prefixes.

TABLE 4: Data updating in each of AP and AR with different global routing prefix, HA, and CN after AMH3 message.

\begin{tabular}{lcc}
\hline AP5 entry table after T2 & AR3 entry routing table after T2 & HA\&CN entry routing table after T2 \\
\hline 2001:1:1:1:1::1/80 + MN-ID temp $(1)$ & 2001:1:1:1:1::1/80 + 2002:1:1:1:1::/80 + MN-ID & 2001:1:1:1:1::1/80 + 2002:1:1:1::/64
\end{tabular}

selected AP which is associated with that visited network. The AMH3 message is included in the association request frame which contains code (11) along with the MN's IPv6 address as shown in Table 1. As mentioned before in AMH1 and $\mathrm{AMH} 2$ the AP creates the MN's ID once the AMH message is received (i.e., AMH3). Afterwards, it forwards AMH3 to the associated AR which includes the MN's IPv6 address, MN's ID, and new AP's IPv6 address. When AR receives the AMH3 message, the default home network IPv6 address that the MN's IPv6 address belongs to is extracted using (AND) process with the unique IPv6 address of MN that was sent via $\mathrm{AMH} 3$ message using the global routing prefix of the MN.

Thus, the NAR which had a different global routing prefix used the extracted home network IPv6 address to send AMH3 through Internet to $\mathrm{HA}$ of MN. The AMH3 message sent by AR included the MN's IPv6 address and the IPv6 address of NAR with a different global routing prefix. Moreover, the IPv6 address of the new AP along with MN's ID is added as a routing entry of the new AR. In other words, the global routing prefix of visited AR is added to the home subnet interface. The AMH3 message was forwarded to $\mathrm{CN}$ by HA in order to inform the new IPv6 address of the newly visited AR to which the $\mathrm{MN}$ is currently connected. Moreover, the IPv6 address of the new AP along with MN's ID is added as a routing entry of the new AR. In other words, the global routing prefix of visited $A R$ is added to the home subnet interface. Thus, the routing table entry of CN was updated by searching MN's IPv6 address then replacing the current active AR's IPv6 address. After the handover was initiated with new selected AP and after the mobility handover has been performed with that selected $\mathrm{AP}$, the previous AP is informed that the MN is physically disconnected after the disassociation frame is invoked by $\mathrm{MN}$ informing AP to canceling its previous session with it; otherwise it will be automatically disassociated by AP after certain timer is over due to no active session is between AP and $\mathrm{MN}$.

On the other hand, after the mobility handover was done with new AR (NAR) the NAR is initiated neighbor advertisement message informing the previous AR of the existence of $\mathrm{MN}$ (MN is currently under its routing table). Thus, the tunnel is established between NAR and previous AR. The process of exchanging AMH3 messages between NAP and ARs continued until the updated AMH3 message reached the $\mathrm{CN}$. These processes occurred during the time point of $T 2$ as illustrated in Figure 6.

In order to shed the light more on the packet flow directions, another scenario of communication is presented that we called down-link communications (CN-to-MN). Therefore, in time point $T 3$ when packets' flow returned from $\mathrm{CN}$ to $\mathrm{MN}$, the packets were encapsulated with the new IPv6 address of the new AR with a different global routing prefix. When AR receives the packet flow which was forwarded to the MN, AR sent it to the new AP's IPv6 address that the MN is currently attached to using the MN's ID that is equipped in an AMH3 message sent by NAP. In order to forward the packet flow by NAP, the MN's ID which was created by NAP is used to send the packets to MN. In other words, the AR and AP used the MN's ID in order to forward the packet flow to the MN instead of the IPv6 address with a different global routing prefix. Thus, the $\mathrm{MN}$ within different subnets and global routing prefixes could keep the proposed unique home IPv6 address without requesting a CoA from each new visited network by using the $\mathrm{AMH} 3$ scheme.

A practical example based on the illustrated scenario in Figure 1 is presented in this paragraph. The updating of the routing table entry data during an $\mathrm{AMH} 3$ process in $\mathrm{AP}, \mathrm{AR}, \mathrm{HA}$, and $\mathrm{CN}$ is shown in Table 4 . When the MN moved from AP4 which belongs to AR2 towards AP5 which belongs to AR3 with a different subnet and global routing 
prefix (2002:1:1:1), the MN sent AMH3 including its IPv6 address with an AMH3 code (11). The AP has included the MN's IPv6 address along with an established MN's ID and its IPv6 address is to be updated as an entry in the AR routing table. By the time the AMH3 message reached HA, the IPv6 address of the $\mathrm{MN}$ has searched its routing table and the current associated AR has been updated from AR2 to AR3 IPv6 address (2002:1:1:1::/64) as shown in Table 4. The same process occurred in $\mathrm{CN}$ when the $\mathrm{AMH} 3$ message from $\mathrm{HA}$ was received.

\section{Performance Evaluation}

4.1. The Numerical Model for AMH. In order to prove the efficiency of the AMH scheme, a numerical model has been created to show the delay time and the cost during handover procedure. In general the mobility handover delay time, $T_{\text {delay, }}$ of $\mathrm{MN}$ includes the handover detection, handover decision, and handover execution delay time. The $T_{\text {delay }}$ can be extracted as in the following form:

$$
T_{\text {delay }}=T_{\text {detection }}+T_{\text {decision }}+T_{\text {execution }}
$$

where $T_{\text {detection }}$ in AMH approach can be calculated as follows:

$$
T_{\text {detection }}=T_{\text {Addr-Config }}+T_{\text {Home-Reg }} .
$$

Formula (6) shows the time taken by $\mathrm{MN}$ in order to detect the next AP. The time of $T_{\text {Addr-Config }}$ and $T_{\text {Home-Reg }}$ is defined as the delay time taken during the BU message exchange between the previous AP and the HA. Since in the AMH scheme the $T_{\text {Addr-Config }}$ and $T_{\text {Home-Reg }}$ have been removed using the unique IPv6 address assigned by HA, the $\mathrm{AMH}$ messaging process is to be performed between the next $\mathrm{AP}$ and next AR and then forwarded through the Internet to the HA. Therefore, the MN informs HA and $\mathrm{CN}$ to upload the attached AR's IPv6 using AMH message while still within the current AP coverage area. Formula (7) shows the handover delay time obtained by utilizing the AMH scheme. Consider

$$
\text { Handover }-T_{\text {delay }}=T_{\text {decision }}+T_{\text {execution }} \text {. }
$$

To analyze the signaling cost in the proposed AMH scheme, the numerical model described below is used.

The total cost in MIPv6 within the same subnet is calculated in Formula (8). Since the IPv6 address of MN remains unchanged within the same subnet during the handover process, the $C_{\text {Add-Config }}$ is eliminated from the signaling cost in inner domain scenario:

$$
C_{\text {Total }}=C_{\text {Detection }}+C_{\text {Add-Config }}+C_{\text {Reg }} \text {. }
$$

On the other hand, Formula (9) calculates the signaling cost in AMH scheme in inner domain. For the duration of the handover within the same subnet, signaling cost includes the mobility detection cost $C_{\text {Detection }}$ and the registration cost $C_{\text {Reg }_{\text {Inner }}}$ which is handled by next AP in AMH scheme using $\mathrm{AMH}$ message sent by $\mathrm{MN}$ to trigger the handover. Hence, the $C_{\text {Reg }}$ haser been eliminated from the $\mathrm{MN}$ side in the proposed AMH scheme as shown in Formula (10). Consider

$$
\begin{gathered}
C_{\text {Inner }}=C_{\text {Detection }}+C_{\text {Reg }} \text { Inner } \\
C_{\text {Inner }}=C_{\text {Detection. }} .
\end{gathered}
$$

In contrast, the signaling cost between different subnets and global routing prefix $C_{\text {Outer }}$ contains the mobility detection cost $C_{\text {Detection }}$, the registration cost $C_{\text {Reg }_{\text {Outer }}}$ between subnets, and the cost of the tunnel between NARs and CARs $C_{\text {Tunnel }}$ as was illustrated in formula (11). In the proposed $\mathrm{AMH}$ scheme, the MN was sending AMH2 and AMH3 employing association request frame to the next $\mathrm{AP}$, and the

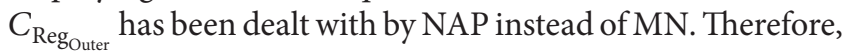
the $C_{\text {Reg }_{\text {Outer }}}$ was omitted from the $\mathrm{MN}$ side in the outer domain as well. Formula (12) shows the calculations of the $\mathrm{C}_{\text {Outer }}$ in AMH scheme. Consider

$$
\begin{gathered}
C_{\text {Outer }}=C_{\text {Detection }}+C_{\text {Reg outer }}+C_{\text {Tannel }}, \\
C_{\text {Outer }}=C_{\text {Detection }}+C_{\text {Tannel }} \text {. }
\end{gathered}
$$

It can be determined from Formulas (10) and (12) that the address configuration cost $C_{\text {Add-Config }}$ which is defined as the cost consumed by the MN to obtain the CoA from NAR and $C_{\text {Reg }}$ to register the change of MN and AR IPv6 address with HA does not exist. This is due to the fact that the $\mathrm{MN}$ is configured with a unique IPv6 address in the proposed AMH scheme without the need to obtain a CoA from visited networks. Moreover, during the handover process the MN informs the newly selected AP through the association request frame to handle the registration process with NAR then forward to HA using AMH messages. Thus, the $C_{\text {Add-Config }}$ and $C_{\text {Reg }}$ in the AMH scheme are eliminated from the MN side which contributes to decreasing the total handover cost.

4.2. Simulation Setup. In this section, we present the simulation setup to validate and evaluate the proposed $\mathrm{AMH}$ scheme. The AMH scheme was modelled and simulated using an INET framework, which is an open-source communication networks simulation package for the OMNeT++ simulation environment. An extensible Mobile IPv6 (xMIPV6) [35] was utilized in order to design and model the proposed AMH scheme that was based on MIPv6. The network layer architectures of xMIPv6 within each of the MN, AP, AR, and HA was modified as a way to maintain the functionality of the proposed AMH scheme during the mobility handover processes.

The TCP/IP used five layers for the design of the network communication architecture for the AMH scheme. The area of the simulation scenario was $2300 \times 1300$ meters and was configured with $\mathrm{OMNeT}++$ simulation based on a map of the Campus of the University of Erlangen-Nuremberg. In addition, the simulation scenario was executed using an Intel(R) Dual-Core CPU $2.40 \mathrm{GHz}$ with OMNeT++ 4.3 simulator software. In the simulated scenario, five different subnets were created that were all based on MIPv6 protocol 


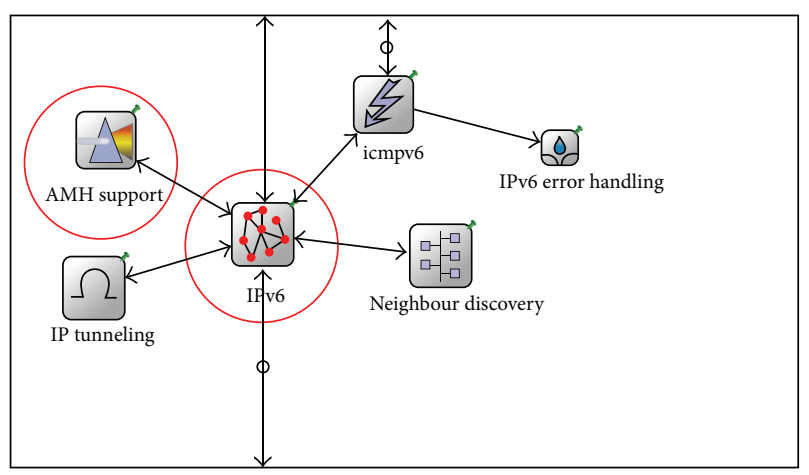

FIgURE 7: Architecture of the AMH scheme network layer.

in the network layer, to evaluate the proposed AMH. Two of these subnets were configured with different global routing prefixes to evaluate the AMH3 subscheme.

In general, each particular subnet was represented by one AR classified as AR1, AR2, or AR3. AR4 and AR5 represented different global routing prefixes used to compare with the other routing prefixes. Two APs were connected to each AR, except for AR5, which was connected with only one AP. Furthermore, each AP function had a transmitted power of $14 \mathrm{dbm}$ and radio coverage that covered 300 meters. The distance between two APs was 500 meters resulting in approximately $20 \%$ of overlapped area. It is worth mentioning that the Internet connections for the ARs were all based on IPv6 addressing.

Figure 7 shows the architectural design of the network layer of the proposed AMH scheme that was designed and configured using OMNeT++. The modified modules are highlighted with red circles. All the AMH supported algorithms were implemented in the AMHsupport module, which was the modified ipv6 module.

Figure 8 shows the underlying design of the link and network layers of the AP, AR, and HA in the simulated scenario. Figure 8(a) presents the architectural design of an AP in the simulated scenario. The highlighted neighbour AP and $\mathrm{MN}-\mathrm{ID}_{\text {temp }}$ table was created in that AP. Figures $8(\mathrm{~b})$ and 8(c) highlight the modified routing tables within AR and HA architecture.

The simulated scenario contains $50 \mathrm{MNs}$, during the first round of simulation they are distributed as follows: Subnet 1, Subnet 2, Subnet 3, and Subnet 4 with $11 \mathrm{MNs}$ in each, while Subnets 5 with 6 MNs. Each MN moves with speed of 1-18 meter/second and starts from the subnet that belongs to which considered as its HA across the other different ARs. The linear and rectangle mobility models were used to generate mobility in the simulated scenario for the AMH scheme. Update intervals of mobility speed that ranged from 0.1 to 1 seconds were configured by the implemented mobility models for each MN. During the roaming process $\mathrm{MN}$ communicates with CNs. The traffic has been generated between $\mathrm{MNs}$ in simulation scenario and $\mathrm{CN}$ as four types of applications HTTP, FTP, VoIP, and video. The traffic model
TABLE 5: The traffic model for HTTP application.

\begin{tabular}{ll}
\hline Parameters & Value \\
\hline Page interarrival times & $\begin{array}{l}\text { Exponentially distributed with } \\
\text { mean } 60 \text { seconds }\end{array}$ \\
& $\begin{array}{l}1000 \text { bytes of text with } 5 \text { medium } \\
\text { images } \\
\text { Page properties }\end{array}$ \\
& $\begin{array}{l}\text { Size randomly picked with a } \\
\text { uniform distribution on }[500,2000] \\
\text { Image properties }\end{array}$ \\
& bytes \\
\hline
\end{tabular}

TABLE 6: The traffic model for FTP application.

\begin{tabular}{lc}
\hline Parameters & Value \\
\hline File transfer & $50 \%$ upload, $50 \%$ download \\
Interrequest file time & Exponentially distributed with \\
mean 720 seconds
\end{tabular}

TABLE 7: The traffic model for voice application.

\begin{tabular}{lc}
\hline Parameters & Value \\
\hline Time spent in silence mode & $\begin{array}{c}\text { Exponentially distributed with } \\
\text { by called and calling party }\end{array}$ \\
Time spent in speech mode & Expoconds \\
by called and calling party & mean 0.352 seconds \\
Voice frame per packet & 1 \\
Bit generation rate & 64 kbps \\
Type of service (ToS) & Best effort (high priority) \\
Compression voice packet & 0.02 second \\
delay &
\end{tabular}

TABLE 8: The traffic model for video application (MPEG-2).

\begin{tabular}{lc}
\hline Frame interval time & 25 frames/sec \\
Frame size (video resolution) & $352 \times 288$ pixels \\
Bit generation rate & 177.4 (low motion) to \\
Bits per pixel & $709.6 \mathrm{kbps}$ (high motion) \\
Type of service (ToS) & 24 \\
\hline
\end{tabular}

for each application runs between MNs and $\mathrm{CN}$ are listed in Tables 5, 6, 7 and 8 .

The MAC layer of the AMH simulation scenario was configured based on IEEE Standard 802.11b, where the PCF was used to simulate the MAC layer of the deployed APs. The channel bandwidth that was used in the simulation was configured to $11 \mathrm{Mbps}$ [36]. In order to set the neighbour discovery process, an interval in the range between the minimum router advertisement interval (minIntervalBetweenRAs $=0.03 \mathrm{~s}$ ) and the maximum router advertisement interval (maxIntervalBetweenRAs $=0.07 \mathrm{~s}$ ) [9] was configured. While the wireless channel settings of WLAN were configured, the passive and active scan processes were activated with a probe delay of 0.1 second and minimum and maximum scanning times of minChannelTime $=0.15$ second and maxChannelTime $=0.3$ second, respectively. The 


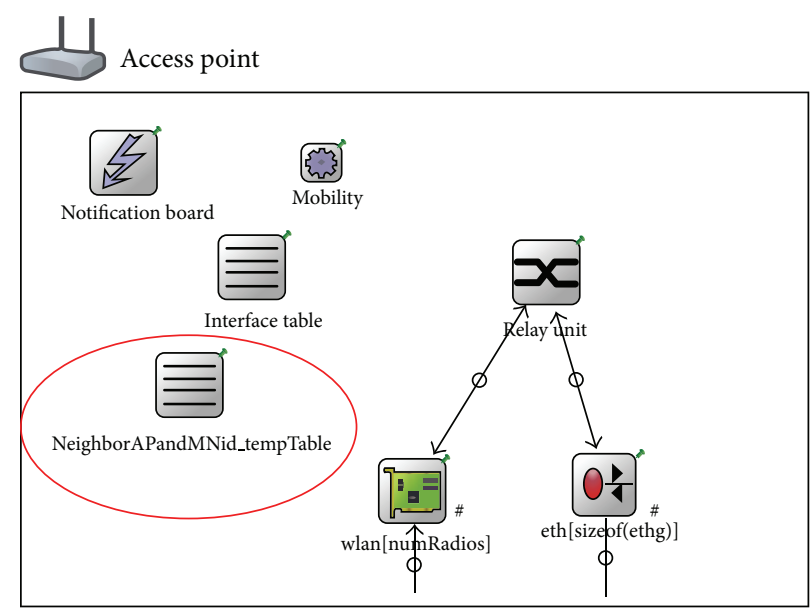

(a) The modified architecture of AP with a list of neighbour AP and MNID temp $_{\text {table }}$

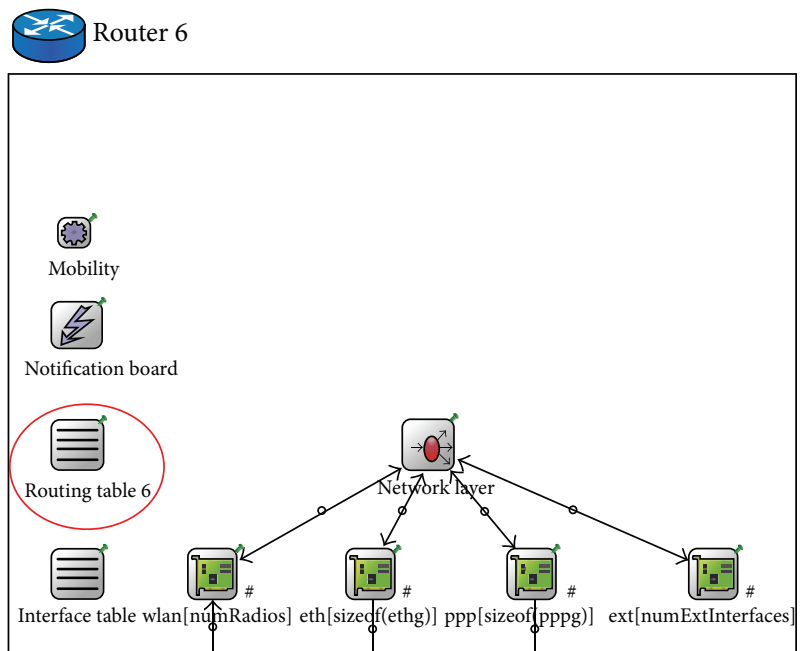

(b) The modified architecture of AR with modified MN routing table format

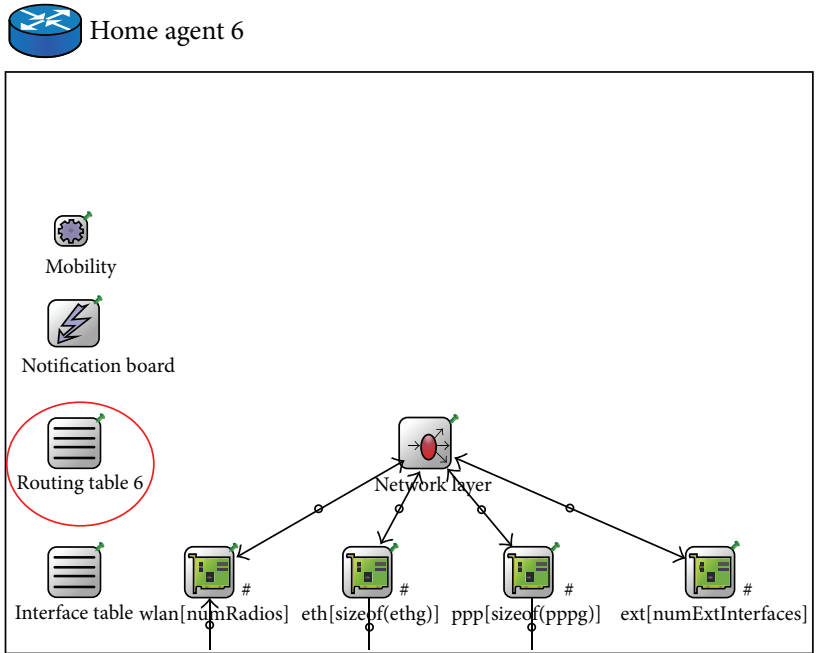

(c) The modified architecture of HA with modified MN routing table format

Figure 8: Architecture design of link and network layers of AP, AR, and HA with modified AMH tables.

authentication and association time-outs were configured to 5 seconds. The maximum size of the higher layer data buffer in bits was specified as 256000 bits.

The maximum packet generation rate set to 800 packets/second with maximum packet size of 1000 bytes in the simulated scenario. The simulation time was set to 700 seconds and the applications start time after 100 seconds of simulation time as a way to avoid the effect of transient behaviour on the simulation results. The simulation parameters are listed as shown in Table 9. Figure 9(b) shows the snapshot of the simulated scenario in OMNeT++.

The received power fluctuates with log-normal distribution about the mean distance-dependent value [37]. The shadowing model is given by

$$
\mathrm{PL}(d)[d B]=\mathrm{PL}\left(d_{0}\right)+10 \times v \times \log \frac{d}{d_{0}}+X_{\sigma},
$$

where $\operatorname{PL}(d)$ is the path loss at distance $d$ between transmitter and receiver, $\operatorname{PL}\left(d_{0}\right)$ is the average path loss at a reference distance is $\left(d_{0}\right), v$ is the path loss exponent, and $X_{\sigma}$ is a zero mean Gaussian distributed random variable with standard deviation $\sigma$.

4.3. Simulation Results. In order to evaluate and validate the proposed AMH scheme, the proposed AMH is compared along with IF-HMIPv6 [28] and SFMMA [29] methods. It is worth mentioning that both the IF-HMIPv6 and SFMMA methods have been discussed earlier in related works section. The performance metrics which are considered to evaluate the proposed AMH scheme and state of the art (IF-HMIPv6 and SFMMA) are total average handover latency during simulation time with (HTTP, FTP, voice, and video) applications, handover signaling overhead cost, binding update signal cost, and the impact of both MN's speed and number on packet loss metric. In order to capture the selected metrics, five MNs have been selected randomly from each particular subnet in a constructed simulation scenario. It should be noted that each of the presented simulation results represents the 
TABLE 9: Simulation parameters.

\begin{tabular}{|c|c|}
\hline Parameters & Value \\
\hline Simulation time & $700 \mathrm{~s}$ \\
\hline Simulation area & $2300 \times 1300 \mathrm{~m}$ \\
\hline Mobility model & $\begin{array}{l}\text { Linear and rectangle } \\
\text { models }\end{array}$ \\
\hline Number of MN & 50 nodes \\
\hline MN speed & $1-18 \mathrm{~m} / \mathrm{s}$ \\
\hline Transmitted power & $14 \mathrm{dbm}$ \\
\hline $\begin{array}{l}\text { Transmission coverage range of each } \\
\text { AP }\end{array}$ & $300 \mathrm{~m}$ \\
\hline Distance between two APs & $500 \mathrm{~m}$ \\
\hline Maximum packet generation rate & $\begin{array}{l}\text { Maximum } 800 \\
\text { packet/second }\end{array}$ \\
\hline Maximum packet size & 1000 byte \\
\hline Channel bandwidth & $11 \mathrm{Mbps}$ \\
\hline MAC protocol & IEEE $802.11 \mathrm{~b}$ \\
\hline
\end{tabular}

average of 10 simulation runs in order to achieve a highly reliable performance evaluation procedure. Moreover, the confidence interval, that is, extremely valuable for any performance evaluation process, arrange of $95 \%$ was configured by utilized ANOVA single factor statistical method. Thus, this confidence interval range is used to estimate the true population value for ANOVA statistic.

4.3.1. Total Handover Latency with HTTP, FTP, Voice, and Video Applications. In order to illustrate the impact of the proposed AMH scheme on the obtained handover latency during the $\mathrm{MN}$ roaming process, the handover latency has been captured with different applications and compared with other proposed methods. Four different kinds of applications are utilized which are categorized as normal network applications (HTTP and FTP) and as real time applications (voice and video). The main point of selecting these four different kinds of traffic is to show the effect of increasing the traffic rate on the handover process with each of the proposed AMH schemes and the state of the art. In other words, when the rate of packets/sec increases this will contribute negatively by increasing the MAC load, channel congestion, and the contention in the channel. Basically, changing traffic type increases the load on the link layer in order to satisfy the traffic requirements. This can be observed especially with real time applications (voice and video) which require a QoS which is normally tackled by MAC of each MN and AP. Therefore, the handover latency has been collected in each of the selected applications with the proposed AMH scheme, IF-HMIPv6, and SFMMA methods.

The total handover latency occurred during simulation time with browsing HTTP application in the proposed AMH scheme, IF-HMIPv6, and SFMMA as illustrated in comparison form in Figure 10(a). The handover latency capture began after 100 seconds and continued until the end of simulation time. The handovers were executed during this time. This is due to the fact that, during the first few seconds of running a simulation process, which is well known as the simulation warm-up process, not all simulation settings have been completed yet. Therefore, in the simulation settings, the capturing results process is configured to begin 100 seconds from the time simulation is executed.

It is clear from Figure 10(a) that the proposed AMH scheme is better able to decrease the total handover latency in comparison with IF-HMIPv6 and SFMMA methods. More precisely, the first time at second 100 when the handover was performed the latency in AMH, IF-HMIPv6, and SFMMA was $(0.06,0.25$, and 0.2 seconds), respectively. It is obvious that the lowest handover latency was obtained using the proposed AMH scheme. Moreover, during the simulation time the handover latency in the AMH scheme ranged between 0.05 and 0.07 seconds which is considered low latency. On the other hand, the obtained latency range was between 0.19 up to 0.25 seconds and 0.09 up to 0.2 seconds in IF-HMIPv6 and SFMMA, respectively.

In contrast, Figure 10(b) depicts the average handover latency obtained each time the handover process occurred between MN and APs while FTP applications were run between $\mathrm{MN}$ and $\mathrm{CN}$. In another words, the handover latency that occurred during a number of handovers performed by the $\mathrm{MN}$ during roaming process has been calculated as an average out of 10 simulation runs with FTP application. Figure 10(b) shows the average handover latency obtained by the $\mathrm{AMH}$ scheme during 5 handovers during simulation time with FTP application. The obtained latency with AMH scheme was $0.19,0.17,0.21,0.26$, and $0.17 \mathrm{Sec}$ during simulation scenario time. The obtained average handover latency during the execution the FTP application with IF-HMIPv6 and SFMMA is $0.79,0.8,0.95,0.99,1.2$, and $1.82 \mathrm{sec}$ and $0.99,1.1,1.65,1.85,1.92$, and $2.1 \mathrm{sec}$, respectively, based on 6 handovers. From the results in Figure 10(b) it can be seen that the handover latency with FTP application increased compared with the HTTP application. This is because the packet rate with FTP application increased compared to the HTTP which contributed to increasing the average handover latency. Furthermore, it can observed that the proposed AMH scheme could save the average obtained handover latency with FTP application, a maximum value of $0.26 \mathrm{sec}$ compared with IF-HMIPv6 and SFMMA 1.82 and $2.1 \mathrm{sec}$, respectively.

Looking at Figure 10(c), the average handover latency obtained when the MN runs on the voice applications with CNs during its roaming process is illustrated. As can be seen, the collected average handover latency is depicted with $(5$, $6,7)$ handover processes performed using AMH scheme, IF-HMIPv6, and SFMMA methods, respectively, with voice application. It is obvious that the handover latency with voice application increased sharply after $100 \mathrm{sec}$ with both IFHMIPv6 and SFMMA during the handover process. However, the AMH scheme could successfully keep the handover latency at an average value that satisfies the voice application requirements. More precisely, by looking at Figure 10 (c) it can be seen that the first handover occurred after 100 seconds with both IF-HMIPv6 and SFMMA methods with delays of 0.99 and $1.09 \mathrm{sec}$, respectively, whereas the obtained delay with the AMH scheme was $0.85 \mathrm{sec}$ after 217 seconds. The 


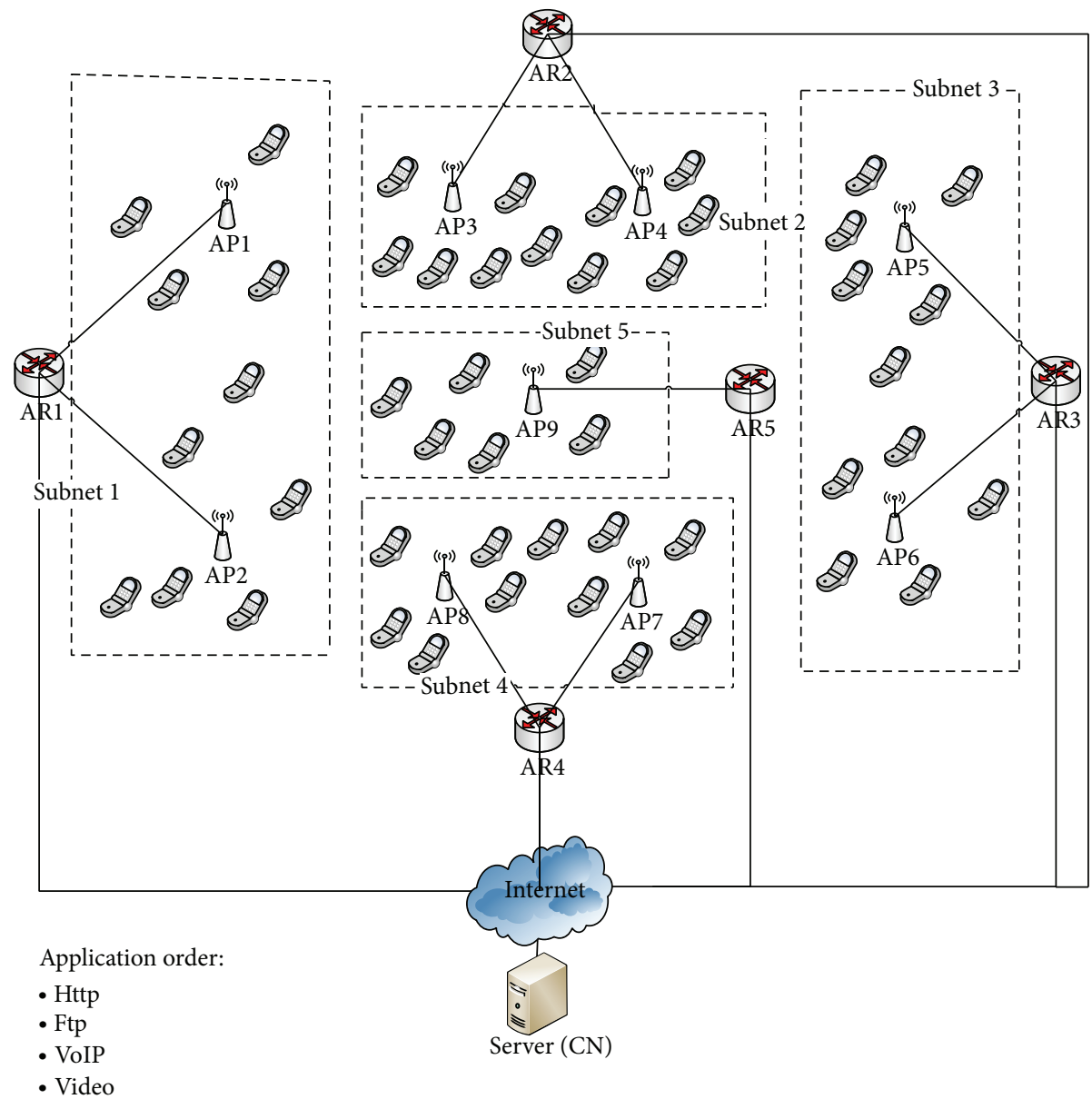

(a) Design of simulation scenario

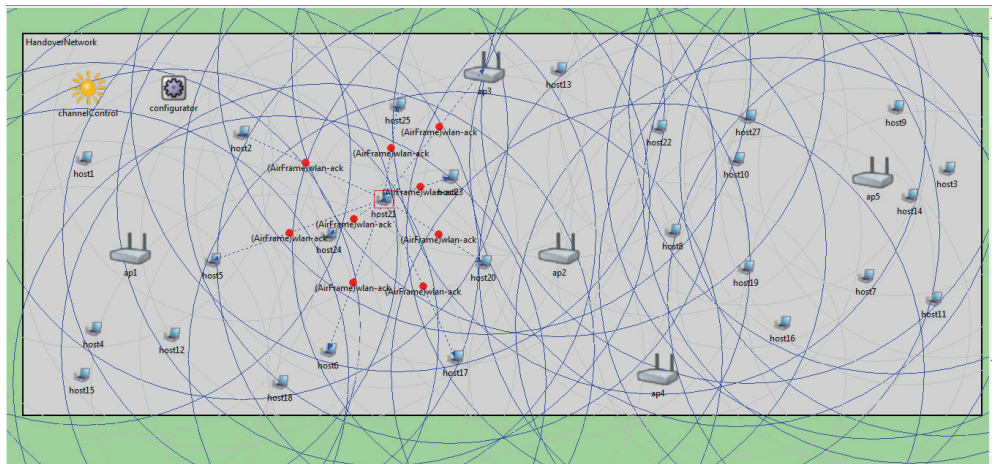

(b) Snapshot of OMNET++ simulation scenario

Figure 9: Simulation scenario.

handover delay kept increasing with both IF-HMIPv6 and SFMMA methods during simulation time rising to $1.3,1.84$, $2.2,2.8$, and 2.82 and $1.50,2.18,2.48,2.85,2.92$, and $3.10 \mathrm{sec}$, respectively.

On the other hand, the handover delay using the proposed AMH scheme continuously deceased to $0.77,0.31,0.26$, and $0.17 \mathrm{sec}$ as shown in Figure 10(c). It is worth mentioning that the proposed $\mathrm{AMH}$ scheme essentially contributes to decreasing the handover delay with voice application compared with IF-HMIPv6 and SFMMA methods. Hence, the QoS voice application requirements performed in the session by $\mathrm{MN}$ are satisfied during the handover process with the AMH scheme with low time delay. Accordingly, the proposed AMH scheme could optimally achieve its objectives, by maintaining a VoIP session with low handover delay compared to the state of the art.

The average handover latency required to execute video applications between $\mathrm{MN}$ and CNs has been captured during simulation time and is illustrated in Figure 10(d). Figure 10(d) shows the obtained handover latency compared to the latency 

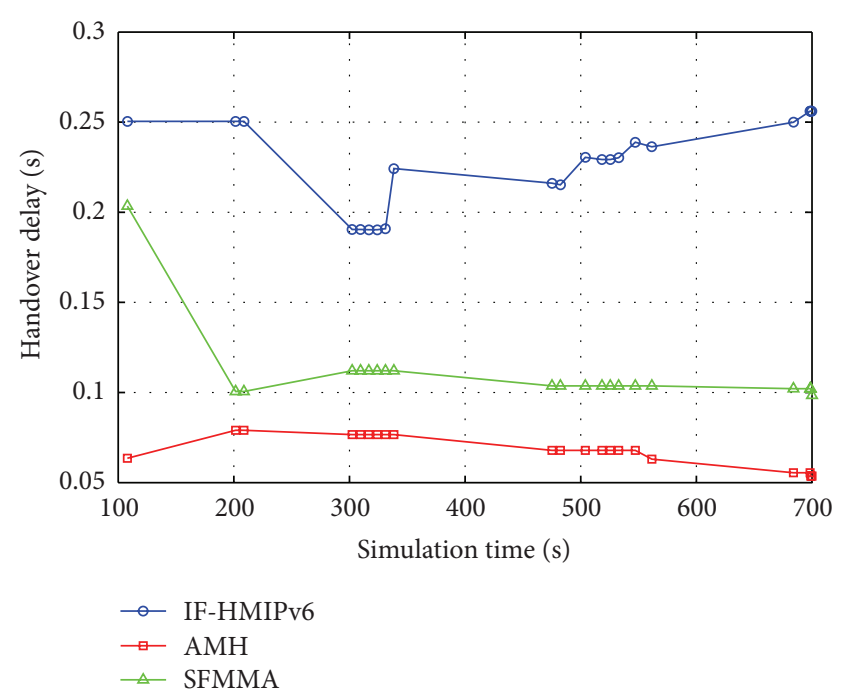

(a) Total handover delay with HTTP application
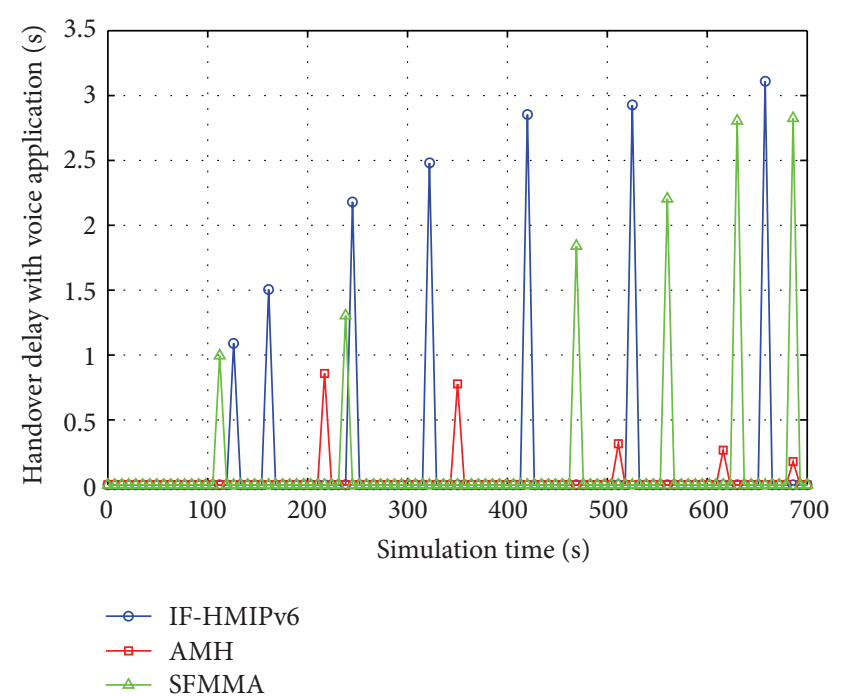

(c) Average handover delay with voice application

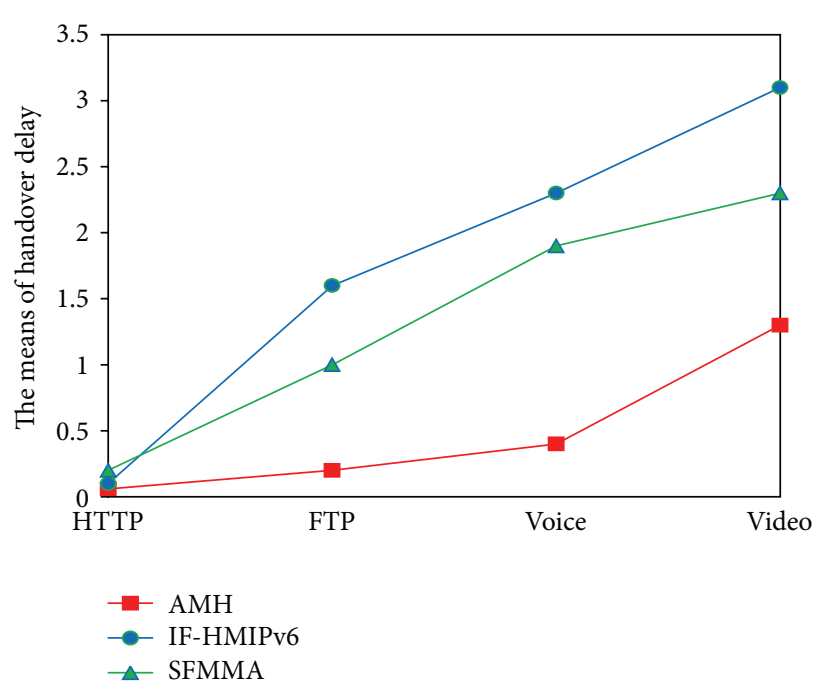

(e) The means of handover delay for HTTP, FTP, voice, and video applications using ANOVA single factor

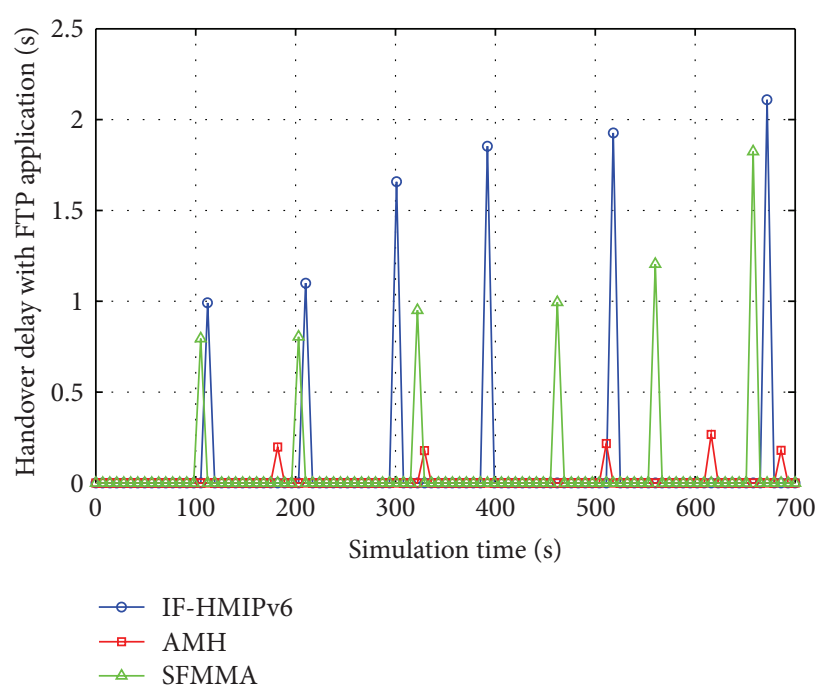

(b) Average handover delay with FTP application
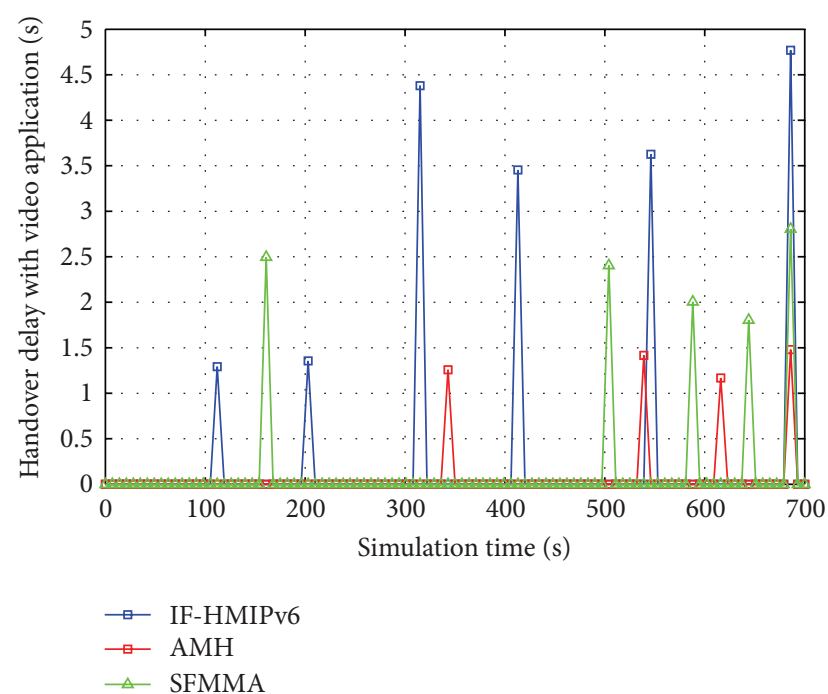

(d) Average handover delay with video application

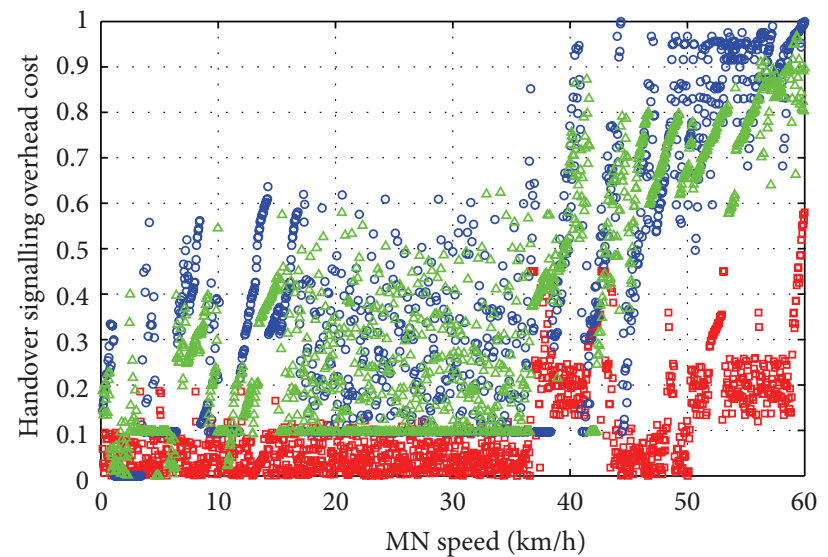

口 $\mathrm{AMH}$

○ IF-HMIPv6

$\triangle$ SFMMA

(f) Normalized handover signalling overhead cost

Figure 10: Continued. 


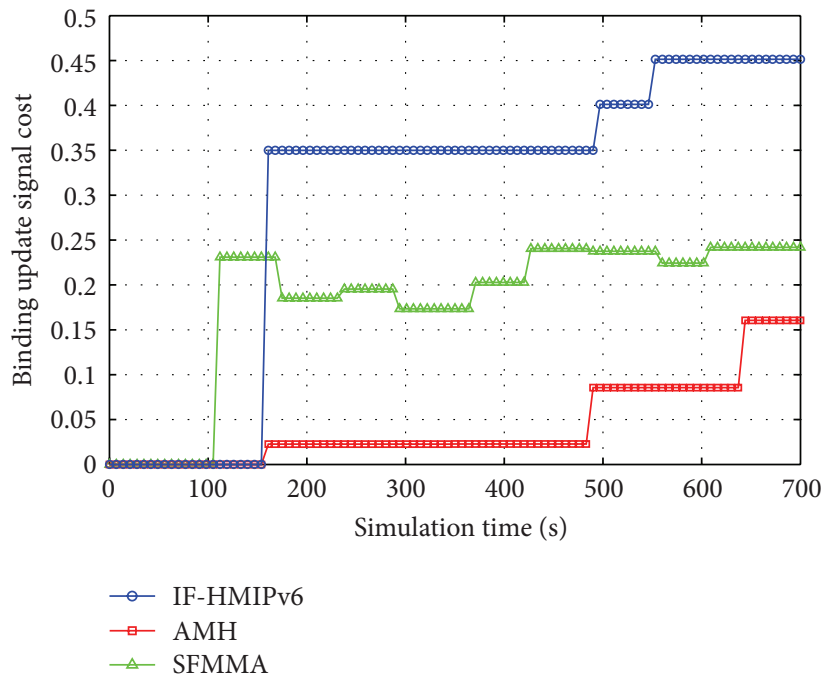

(g) Binding update signal cost

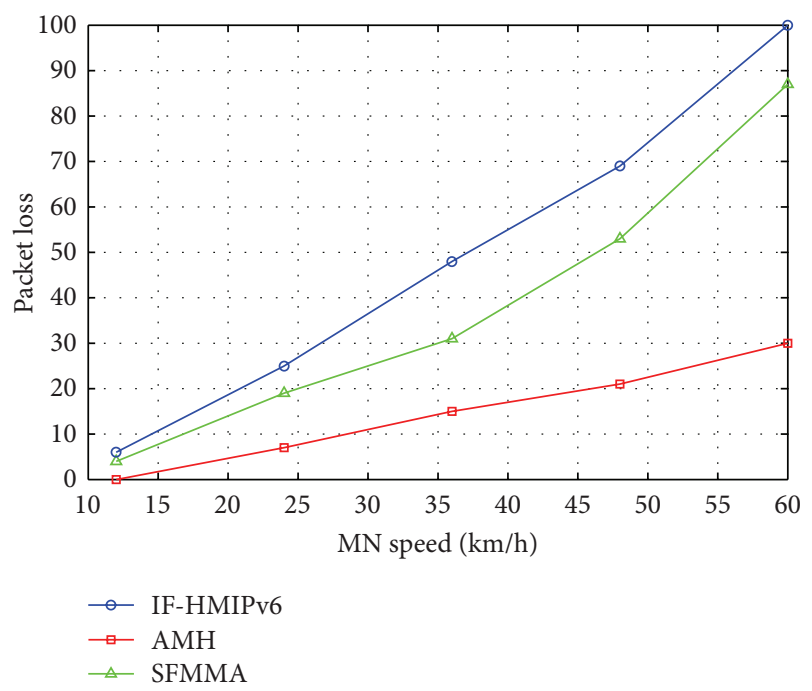

(h) Impact of MN's speed on packet loss

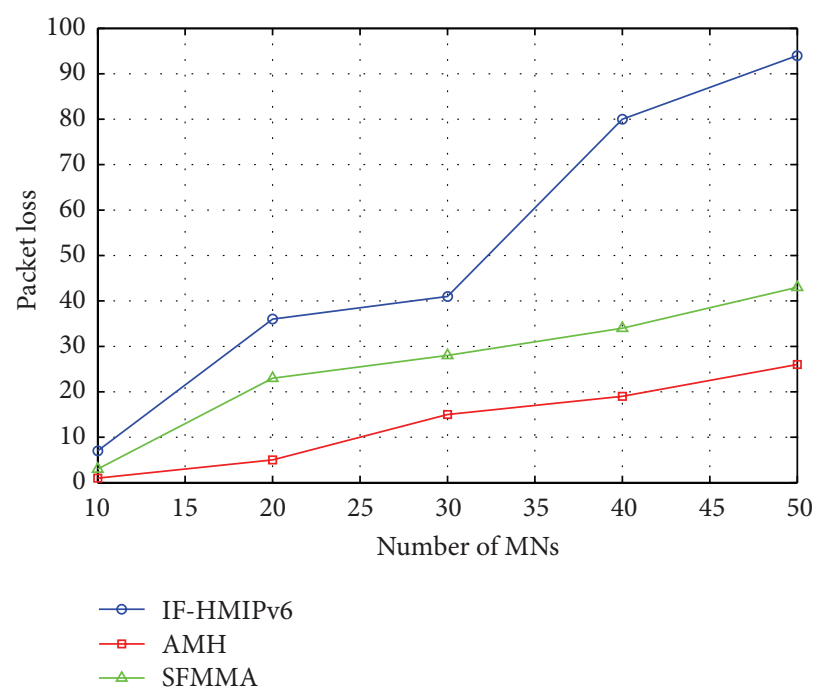

(i) Impact of MN's number on packet loss

Figure 10: AMH evaluation metrics.

of the AMH scheme, IF-HMIPv6, and SFMMA. It can be observed that the MN using the proposed $\mathrm{AMH}$ scheme tackled 4 times the handover process with delays of $1.25,1.41$, 1.16 , and $1.47 \mathrm{sec}$ at the simulation time $343,539,616$, and $686 \mathrm{sec}$, respectively. On the other hand, the IF-HMIPv6 and SFMMA methods MN handled 5 and 6 times the handover process with delay times of $2.49,2.4,2,1.8$, and 2.8 and 1.29 , $1.35,4.38,3.45,3.62$, and $4.76 \mathrm{sec}$ at the simulation time 161 , $504,588,644$, and 686 and 112, 203, 315, 413, 546, and $686 \mathrm{sec}$, respectively.

As can be seen from the results in Figure 10(d), the handover delay during simulation time was obviously not constant especially with SFMMA method with high handover delay compared with the AMH scheme and IF-HMIPv6. This is due to the fact that the handover process with video traffic is considered a heavy real time application which consequently has a negative effect on the obtained handover delay. In other words, when the packet rate transferred per second increased each time the $\mathrm{MN}$ sends or receives video traffic with $\mathrm{CN}$ during the time a handover process is performed by the MN, the handover latency increased due to the MAC load which was mentioned earlier in this section. Moreover, the MN in SFMMA method relies on LMA to search for a possible candidate to direct this traffic flow in addition to the existing time delay of configuring the home network prefixes (HNP) between MN, MAG, and LMA which increases the total handover delay.

On the contrary, the obtained handover delay using IFHMIPv6 method during video application was high compared with the proposed AMH scheme in spite of introducing a new way to formulate the new CoA in the MAP. This is because the CoA registration process is still visible in the MAP side during MN roaming in addition to the time consumed waiting to obtain the new CoA from the $\mathrm{MN}$ which 
is still valid as the IPv6 address of the MN changes when visiting different subnets. Due to the aforementioned reasons, the obtained handover delay with video application using both IF-HMIPv6 and SFMMA methods increased compared with the proposed AMH scheme.

In order to validate the presented handover delay results, ANOVA single factor method was utilized. Figure 10(e) compares the average handover delay of AMH, SFMMA, and IFHMIPv6 using ANOVA single factor for HTTP, FTP, voice, and video applications. The result indicates that AMH has the lowest means compared with the other two approaches. The illustrated validation results indicate that the means of AMH, SFMMA, and IF-HMIPv6 are 0.06, 0.1, and $0.2 \mathrm{sec}$, respectively, for HTTP application with $F$ value of 372.47 and $p$ less than $1 \%$ level of significance. In contrast, the means of handover delay for FTP application of AMH, SFMMA, and IF-HMIPv6 are $0.2,1$, and 1.6, respectively, with $F$ value of 20.88 and $p$ less than $1 \%$ level of significance. The obtained means of handover delay for voice application of $\mathrm{AMH}, \mathrm{SFMMA}$, and IF-HMIPv6 are 0.4, 1.9, and $2.3 \mathrm{sec}$, respectively, with $F$ value of 11.66 and $p$ less than $1 \%$ level of significance.

Finally, the means of handover delay for video application of AMH, SFMMA, and IF-HMIPv6 are 1.3, 2.3, and $3.1 \mathrm{sec}$, respectively, with $F$ value of 4.03 and $p$ less than 5\% level of significance. These results suggest that the AMH scheme has lower means than the other two methods. The implication is that the method may be more efficient in reducing handover delay in HTTP, FTP, voice, and video applications than the other two methods as shown in Figure 10(e). Thus, the applied ANOVA single factor validation method significantly reflects the credibility of the proposed AMH scheme.

Consequently, it can be concluded from the presented handover latency results of HTTP, FTP, voice, and video applications that the AMH scheme could achieve the lowest handover delay with each particular application compared with the other proposed methods. This is no surprise since the proposed AMH scheme utilizes an advanced mobility model with a unique IPv6 address. There are two main advantages achieved by the AMH scheme. First, the ability to eliminate the time delay spent in obtaining a new CoA each time an $\mathrm{MN}$ visits a new network with a different subnet. To this must be added the time consumed in performing the handover in order to change the newly obtained AR's IPv6 address in each HA and CN routing entry. Thus, instead of the MN engaging during handover process by obtaining a new CoA and informing the HA and $\mathrm{CN}$ of the change in IPv6 address after the link layer handover which causes high handover latency, NAP tackles the registration process with a unique IPv6 address. Hence, the AMH scheme in general contributes essentially to decreasing the total handover delay.

4.3.2. Handover Signalling Cost. In order to investigate the performance of AMH scheme in terms of reducing the cost of signaling overhead of handover procedure, in this section the results of total signaling cost and $\mathrm{BU}$ cost are presented and discussed. Figure 10(f) illustrates the impact of MN's speed on the cost of handover signaling overhead of handovers which are performed employing AMH scheme, IF-HMIPv6, and SFMMA methods during simulation time. Normally, during handover process a location update procedure is performed which consists of several costs such as the binding update cost generated due to MNs mobility and the periodic binding refresh cost generated due to the expiration of the binding lifetime. By increasing the movement speed, the time required to process the aforementioned mobility handover process will be increased which subsequently increases the total signals overhead. For this reason, the impact of MN's speed on mobility handover signaling overhead is investigated in this section.

It can be observed from the results presented in Figure 10(f) the behaviour of signaling cost of performed handovers which was increasing similarly to MN's speed. Obviously, the proposed AMH scheme performed the best by saving the cost of signals which are associated with handover process in low average cost by the time the $\mathrm{MN}$ increases the movement speed compared with the state of the art. Regrettably, the average handover signaling overhead cost is the highest utilizing IF-HMIPv6 method which is increasing similar to the speed. In contrast, it can be observed that SFMMA method performed better than IF-HMIPv6 by obtaining lower signaling overhead cost.

The reason behind this is that the time the $\mathrm{MN}$ requires to perform more signals with other network components (MAP, MAG, and LMA) leads to establish a lifetime parameter for every particular signal; when times expires, the signal must be resent once again. In other words, with every handover signal performed there is a lifetime associated whereby decreasing this time as a way to avoid higher delay results in rising signal refreshment costs. Therefore, the proposed AMH scheme significantly decreased the number of signals which are triggered by the $\mathrm{MN}$ side to be combined into one developed AMH message. To this end, this dictated the tremendous improvement in decreasing the total handover signaling overhead cost using proposed AMH scheme.

On the other hand, the cost of binding update signal messages is collected employing the AMH scheme, IFHMIPv6, and SFMMA methods as presented in Figure 10(g). Specificity, the cost of a BU signal message is calculated during simulation time at the time handovers which are performed by MN with APs in the simulated area. It is obvious that by the time handovers started after $100 \mathrm{sec}$ of simulation time the BU cost started increasing slightly using the AMH scheme in contrast to the state of the art which sharply increased. As mentioned earlier in this section, when the $\mathrm{MN}$ is engaged with the $\mathrm{BU}$ signal message process it takes on the responsibility of the $\mathrm{BU}$ refreshing process which was performed due to the expiration of the binding lifetime. Hence, the obtained BU cost using IF-HMIPv6 is the highest followed by SFMMA method which utilizes media independent handover function (MIHF).

The implementation of MIHF of IEEE 802.21 standard in SFMMA method provides more improvement in the handover process compared with IF-HMIPv6 which applies the concept of HMIPv6 protocol. Although MIHF defines three different services, media independent event service (MIES), media independent command service (MICS), and media independent information service (MIIS), the MN 
is still associated with the network link changing process when the handover is triggered by the MN itself in SFMMA method. In contrast, the proposed AMH scheme omitted any interaction between the $\mathrm{MN}$ and any of the network components, except triggering the development of the $\mathrm{AMH}$ message which is embedded into the association request frame from the MN to selected APs.

4.3.3. Packet Loss. The packet loss performance metric is investigated and evaluated in this section as a way of identifying the level of improvement that was achieved by decreasing the disconnect time due to the handover process employing the proposed $\mathrm{AMH}$ scheme. In order to achieve an accurate packet loss metric evaluation, the impact of MN's speed and its number are collected and presented in Figures 10(h) and 10(i), respectively. Figure 10(h) presents the packet loss by the time the $M N$ increases its speed $(\mathrm{km} / \mathrm{h})$. It is obvious from the graph of packet loss's behaviour that at the initial MN's speed, the packet loss obtained by operating the AMH scheme is equal to zero, and almost the same for both IFHMIPv6 and SFMMA methods with low loss ratio. In this regard, it can be said that the $\mathrm{MN}$ with low speed has adequate time to establish the new connection with selected APs and to redirect the traffic flow of the MN to NAP. Therefore, the packet loss experienced a delay at $12 \mathrm{~km} / \mathrm{h}$ which is the time required to send $\mathrm{BU}$ to the $\mathrm{HA}$ which is already eliminated in the proposed $\mathrm{AMH}$ scheme by elaborating the developed AMH message. Thus, the AMH scheme initially obtained zero packet loss compared to the state of the art.

As the speed increases, the packet loss ratio increased up to a maximum at $60 \mathrm{~km} / \mathrm{h}$ speed using IF-HMIPv6 followed by SFMMA method compared to the proposed AMH scheme. AMH performed the best by efficiently decreasing the packet loss ratio. The important point to note here is that the time which is available to complete the processes of obtaining CoA, registration, and BU procedures was shorter as the MN's speed increases. Subsequently, a greater packet loss ratio was experienced as the MN speed was increasing. An additional weakness associated with increasing MN's speed is that the default nature of AP's channel accessibility decreases in WLANs based on IEEE 802.11b due to contention which occurs as speed increases. Accordingly, it can be possible that the MN does not obtain access over the wireless channel of the AP immediately after sending the association request frame; if not, it will be delayed. Regrettably, at higher speeds, wireless access contention increases, resulting in packet loss. In spite of the aforementioned high speed consequences, the AMH scheme could achieve lower packet loss ratio, compared to the state of the art, by keeping the value of packet loss at acceptable levels with low handover delay.

On the other hand, Figure 10(i) depicts the packet loss observed for a different number of MNs at a constant speed of 5 meters per second. When the handover is performed at a constant, low speed, the MN has sufficient time to execute CoA obtaining and registration processes. This implies that packet loss arises only during the time that the sending $\mathrm{BU}$ procedure to the HA is processed. Therefore, proportionally, when the number of MNs increases, packet loss increases due to the time delay experienced by the BU packet in order to reach its HA with a high number of MNs performing handovers.

In light of the above discussion, it can be observed that the AMH scheme can efficiently decrease the packet loss ratio as the number of MNs increases. In other words, with a maximum of $50 \mathrm{MNs}$, the AMH scheme obtained an average packet loss of 26 packets; the loss was 94 and 43 packets using IF-HMIPv6 and SFMMA methods, respectively. The reason is that the proposed scheme efficiently decreased the mobility handover delay by introducing developed AMH messages (AMH1: within the same subnet; AMH2: within different subnets with the same global routing prefix; and AMH3: within different subnets and different global routing prefixes). These AMH messages are devised to perform mobility handovers in advance of the handover in the link layer which suspends the high delay associated with the BU procedure. In addition, proposing unique IPv6 addresses to be utilized by MIPv6 protocols which are configured to all MNs functioning under the AMH scheme omits the procedures which belong to the CoA process. To this end, the AMH scheme efficiently decreased the packet loss ratio with respect to two impacts, MN's speed, and number compared to the state of the art.

\section{Comparison of Main Handover Protocols Features}

A critical comparison in terms of certain parameters is conducted between the well known mobility management handover protocols and presented by Table 10. It is clear that our proposed AMH scheme could achieve the best rating in terms of improving the main features in mobility-based management protocols. The main point to highlight here is that our proposed AMH scheme could efficiently decrease the mobility handover delay by elaborating an advanced handover in addition to the developed unique IPv6 address. Thus, the signalling cost was reduced; afterwards it was reflected as an improvement achieved with packet loss of running applications.

\section{Conclusion}

In this paper, we proposed an advanced mobility handover scheme to perform the network layer handover prior to its normal time (before the handover in link layer has been processed). These AMH scheme's processes were carried out by the next AP (NAP) after the MN triggers the developed AMH message that was embedded with association req $_{\text {frame }}$ during the association process with NAP. Thus, the handover in the network layer level was performed by NAP on behalf of the MN while it was still communicating with the current AP. Moreover, a unique IPv6 address was developed in this paper as a way to keep the IPv6 address of the MNs during the simulated scenarios unchanged. In other words, during the time the MNs were visiting other wireless network providers with different subnets or different global routing prefixes, the MNs were not required to obtain a CoA from the visited networks. This was achieved by creating three main fields 
TABle 10: Comparison Between Main Mobility Management Protocols and Proposed AMH Scheme.

\begin{tabular}{|c|c|c|c|c|c|}
\hline Parameters & MIPv6 & HMIPv6 & FMIPv6 & PMIPv6 & Proposed AMH \\
\hline Complexity & Medium & High & High & Medium & Low \\
\hline Handover Delay & High & High & Low & Medium & Very Low \\
\hline Scalability & Medium & Medium & Medium & Low & High \\
\hline Packet Loss & High & Medium & Medium & Medium & Low \\
\hline Mobility & Host-Based & Host-Based & Host-Based & Network-Based & Host-Based \\
\hline Signalling Overhead & High & Medium & High & Medium & Low \\
\hline Operating Layer & Network Layer & Network Layer & Network Layer & Network Layer & Link and Network Layers \\
\hline Mobility scope & Global & Local & Global/(local) & Local & Global/(local) \\
\hline Handover Management & Yes (limited) & Yes & Yes & Yes & Yes \\
\hline Location Management & Yes & Yes & No & Yes & No \\
\hline Required Infrastructure & $\mathrm{HA}$ & HA, MAP & HA, optimised AR & LMA, MAG & HA, optimised AP, AR \\
\hline Modification on MN & Yes & Yes & Yes & - & Yes \\
\hline Route Optimization & Yes & Yes & - & - & Yes \\
\hline Movement Detection & Required & Required & Required & Performed by L2 & Performed by L2 \\
\hline
\end{tabular}

in IPv6 address format, which are global routing prefix, APID, and MN-ID fields. Furthermore, three tables identified as neighbour $\mathrm{AP}$ and $\mathrm{MN}-\mathrm{ID}_{\text {temp }}$ tables in $\mathrm{APs}, \mathrm{MN}$ routing table in ARs, and Local MN table in HAs were cached. Employing these tables, routing and MN's IPv6 address registration processes became more dynamic in updating the ARs' IPv6 address during MN roaming. Therefore, numerical analysis has proven that by using the proposed AMH scheme, many of the costs and time delays that were normally associated to the handover process have been suppressed. Extensive and fair simulation results show that, compared to the other proposed methods IF-HMIPv6 and SFMMA, our AMH scheme performed better in terms of total and average handover latency with (HTTP, FTP, voice, and video) applications. Moreover, in terms of handover signaling overhead cost, binding update signal cost, and the impact of MN's speed in addition to the number on packet loss metric our AMH scheme performed the best. We are currently working on development of a prediction algorithm to reduce the handover latency in heterogeneous wireless networks.

\section{Conflict of Interests}

The authors declare that there is no conflict of interests regarding the publication of this paper.

\section{References}

[1] I. You, Y.-H. Han, Y.-S. Chen, and H.-C. Chao, "Next generation mobility management," Wireless Communications and Mobile Computing, vol. 11, no. 4, pp. 443-445, 2011.

[2] A. Canovas, D. Bri, S. Sendra, and J. Lloret, "Vertical WLAN handover algorithm and protocol to improve the IPTV QoS of the end user," in Proceedings of the IEEE International Conference on Communications (ICC '12), pp. 1901-1905, Ottawa, Canada, June 2012.
[3] L. Li, L. Ma, Y. Xu, and Y. Fu, "Motion adaptive vertical handoff in cellular/WLAN heterogeneous wireless network," The Scientific World Journal, vol. 2014, Article ID 341038, 7 pages, 2014.

[4] K. Nahrstedt, "Quality of servce in wireless networks over unliensed spectrum," Synthesis Lectures on Mobile and Pervasive Computing, vol. 6, no. 1, pp. 1-176, 2011.

[5] I. Cho, K. Okamura, T. W. Kim, and C. S. Hong, "Performance analysis of IP mobility with multiple care-of addresses in heterogeneous wireless networks," Wireless Networks, vol. 19, no. 6, pp. 1375-1386, 2013.

[6] L. A. Magagula, H. A. Chan, and O. E. Falowo, "Handover approaches for seamless mobility management in next generation wireless networks," Wireless Communications and Mobile Computing, vol. 12, no. 16, pp. 1414-1428, 2012.

[7] A. S. Sadiq, K. A. Bakar, K. Z. Ghafoor, J. Lloret, and S. Mirjalili, "A smart handover prediction system based on curve fitting model for Fast Mobile IPv6 in wireless networks," International Journal of Communication Systems, vol. 27, no. 7, pp. 969-990, 2014.

[8] A. S. Sadiq, K. Abu Bakar, K. Z. Ghafoor, J. Lloret, and R. Khokhar, "An intelligent vertical handover scheme for audio and video streaming in heterogeneous vehicular networks," Mobile Networks and Applications, vol. 18, no. 6, pp. 879-895, 2013.

[9] D. Johnson, C. Perkins, J. Arkko et al., "Rfc 3775: Mobility support in ipv6," Nokia Research Center, June 2004.

[10] A. S. Sadiq, K. Abu Bakar, K. Z. Ghafoor, and J. Lloret, "Intelligent vertical handover for heterogeneous wireless network," in Proceedings of the World Congress on Engineering and Computer Science, vol. 2, pp. 774-779, San Francisco, Calif, USA, October 2013.

[11] C. Perkins, “Rfc 3344: Ip mobility support for ipv4,” Network Working Group, 2002.

[12] D. Johnson, C. Perkins, and J. Arkko, "Rfc 3775: mobility support in ipv6, obsoletes," July 2011.

[13] H. Soliman, L. Bellier, and K. E. Malki, "Rfc 5380: hierarchical mobile ipv6 mobility management (hmipv6)," Network Working Group, 2008. 
[14] H. J. Jang, Y. H. Han, J. Jee, S. D. Park, and J. Cha, "Rfc: 5270 mobile ipv6 fast handovers over ieee 802.16e networks," Network Working Group, 2008.

[15] K.-W. Lee, W.-K. Seo, Y.-Z. Cho, J.-W. Kim, J.-S. Park, and B.$S$. Moon, "Inter-domain handover scheme using an intermediate mobile access gateway for seamless service in vehicular networks," International Journal of Communication Systems, vol. 23, no. 9-10, pp. 1127-1144, 2010.

[16] C.-W. Lee, M. C. Chen, and Y. S. Sun, "Protocol and architecture supports for network mobility with QoS-handover for highvelocity vehicles," Wireless Networks, vol. 19, no. 5, pp. 811-830, 2013.

[17] C. Castelluccia, "Hmipv6: a hierarchical mobile ipv6 proposal," ACM SIGMOBILE Mobile Computing and Communication Review, vol. 4, no. 1, pp. 48-59, 2000.

[18] M. H. Masud, F. Anwar, S. M. S. Bari, and O. M. Mohamed, "Enhancement of handoff latency reduction mechanism of Mobile Internet Protocol version 6 (MIPv6)," in Proceedings of the International Conference on Computer and Communication Engineering (ICCCE '12), pp. 700-705, IEEE, Kuala Lumpur, Malaysia, July 2012.

[19] J. Kempf, "Rfc 4830: problem statement for network-based localized mobility management (netlmm)," Network Working Group, 2007.

[20] R. Koodli, “Rfc 5268: Mobile ipv6 fast handovers," IETF, June 2009.

[21] V. Vassiliou and Z. Zinonos, "An analysis of the handover latency components in mobile IPv6," Journal of Internet Engineering, vol. 3, no. 1, 2010.

[22] S. Gundavelli, K. Leung, V. Devarapalli, K. Chowdhury, and B. Patil, "Rfc 5213: proxy mobile ipv6," IETF, August 2008.

[23] G. Giaretta, $R f c$ 6612: Interactions between Proxy Mobile ipv6 (pmipv6) and Mobile ipv6 (mipv6), IETF, 2012.

[24] H. Modares, A. Moravejosharieh, J. Lloret, and R. B. Salleh, “ survey on proxy mobile ipv6 handover," IEEE Systems Journal, 2014.

[25] L. Wang, M. Song, and J.-D. Song, "An efficient hierarchical authentication scheme in mobile IPv6 networks," The Journal of China Universities of Posts and Telecommunications, vol. 15, pp. 9-13, 2008.

[26] H. Modares, A. Moravejosharieh, J. Lloret, and R. Salleh, "A survey of secure protocols in mobile IPv6," Journal of Network and Computer Applications, vol. 39, no. 1, pp. 351-368, 2014.

[27] H. Modares, R. B. Salleh, A. Moravejosharieh, and J. Lloret, "Enhancing security in mobile IPv6," ETRI Journal, vol. 36, no. 1, pp. 51-61, 2014.

[28] C.-W. Wu and P. Wang, "Improved fast handover scheme for hierarchical mobile IPv6," in Proceedings of the 4th International Conference on Computer Science and Education (ICCSE '09), pp. 294-297, IEEE, July 2009.

[29] R. I. Meneguette, L. F. Bittencourt, and E. R. M. Madeira, "A seamless flow mobility management architecture for vehicular communication networks," Journal of Communications and Networks, vol. 15, no. 2, pp. 207-216, 2013.

[30] I. Al-Surmi, M. Othman, N. A. W. Abdul Hamid, and B. M. Ali, "Enhancing inter-PMIPv6-domain for superior handover performance across IP-based wireless domain networks," Wireless Networks, vol. 19, no. 6, pp. 1317-1336, 2013.

[31] R. M. Hinden and B. Haberman, "Rfc 4193: unique local ipv6 unicast addresses," IETF, 2005.
[32] M. Wasserman and F. Baker, "Rfc 6296: Ipv6-to-ipv6 network prefix translation," Internet Engineering Task Force (IETF), 2011.

[33] D. E. Eastlake, J. I. Schiller, and S. Crocker, "Rfc 4086: randomness requirements for security," Network Working Group, 2005.

[34] IEEE Computer Society, "Wireless lan medium access control (mac) and physical layer specifications," 2007, http://standards .ieee.org/getieee802/802.11.html.

[35] F. Z. Yousaf, C. Bauer, and C. Wietfeld, "An accurate and extensible mobile ipv6 (xmipv6) simulation model for omnet ,$++ "$ in Proceedings of the 1st International Conference on Simulation Tools and Techniques for Communications, Networks and Systems \& Workshops, ICST (Institute for Computer Sciences, Social-Informatics and Telecommunications Engineering), ACM Digital Library, p. 88, Marseille, France, March 2008.

[36] WLAN-MAC, "Wireless lan medium access control (mac) and physical layer specifications," IEEE Computer Society, 2007, http://standards.ieee.org/getieee802/802.11.html.

[37] T. S. Rappaport, Wireless Communications: Principles and Practice, Prentice Hall, 2nd edition, 2002. 

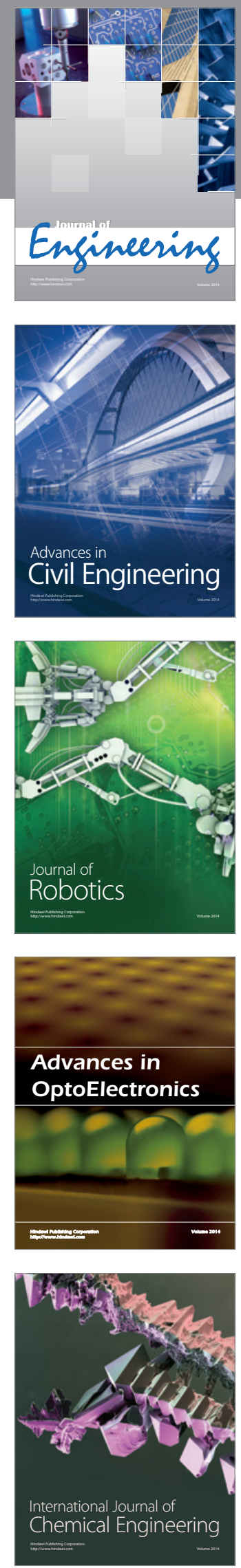

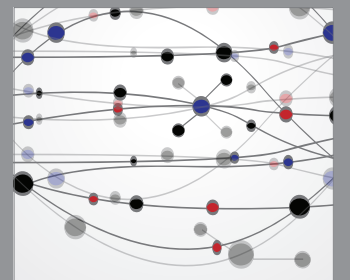

The Scientific World Journal
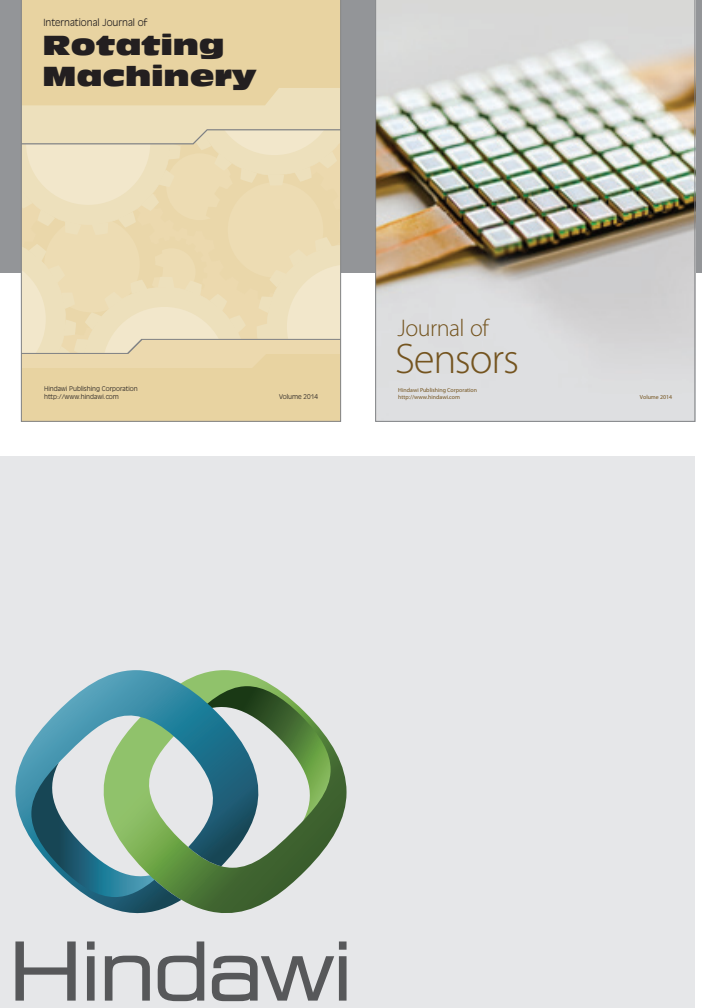

Submit your manuscripts at http://www.hindawi.com
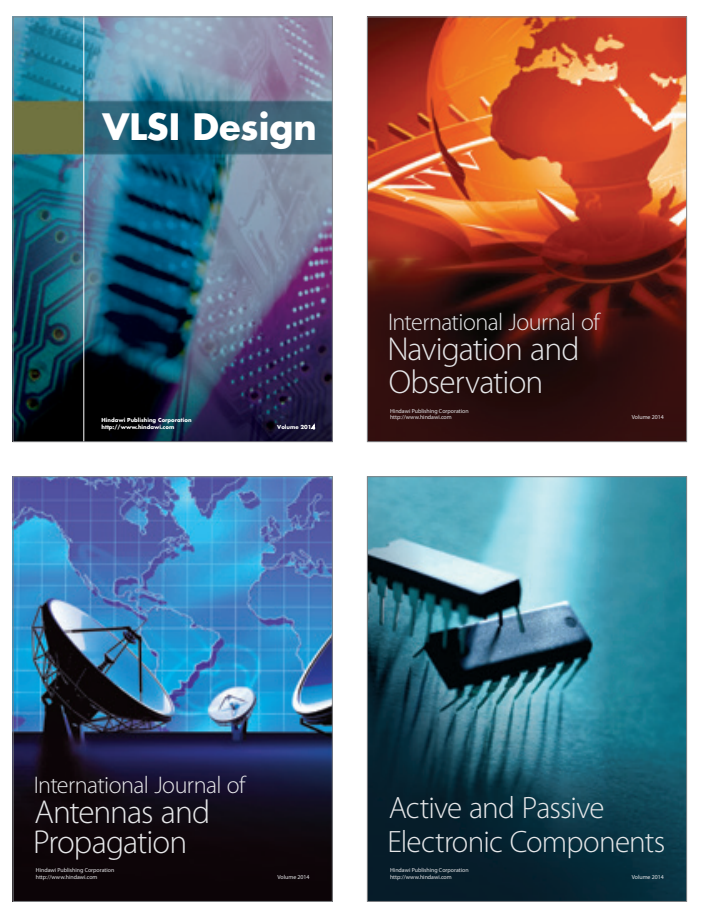
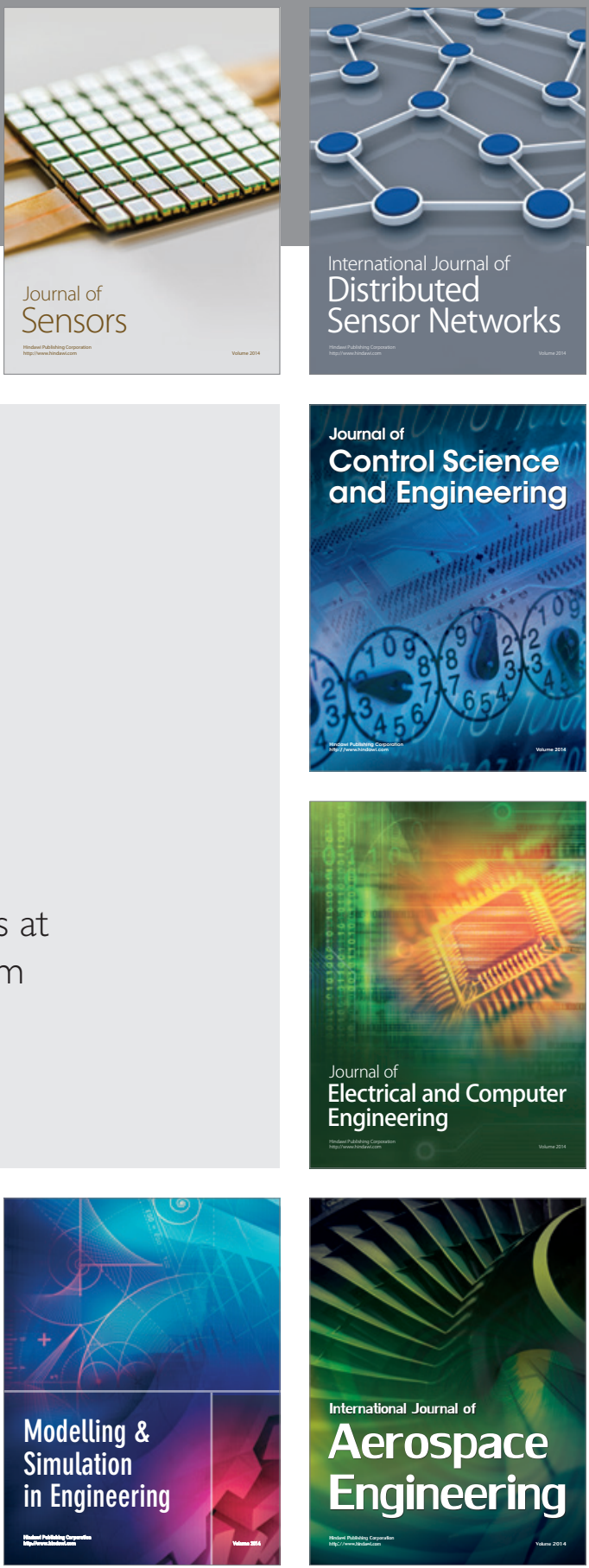

Journal of

Control Science

and Engineering
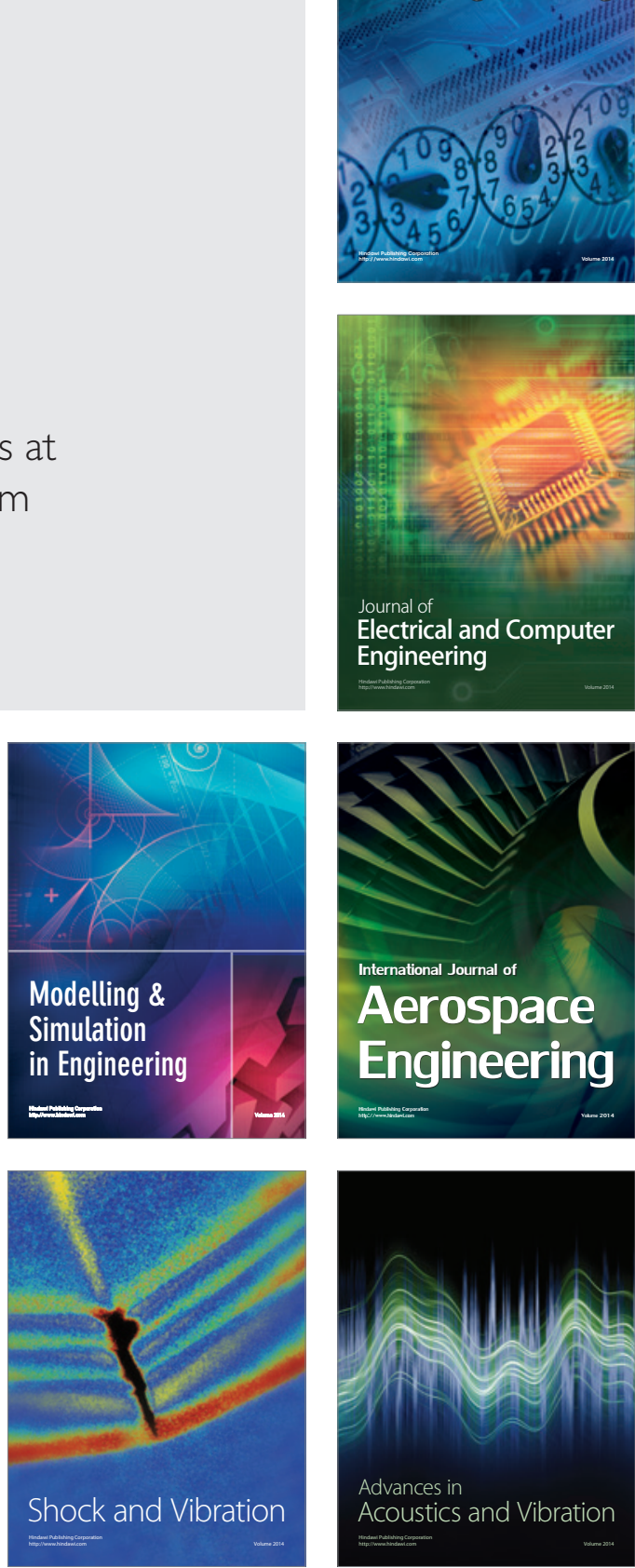